\title{
Experimental properties of gluon and quark jets from a point source
}

The OPAL Collaboration

G. Abbiendi ${ }^{2}$, K. Ackerstaff ${ }^{8}$, G. Alexander ${ }^{23}$, J. Allison ${ }^{16}$, N. Altekamp ${ }^{5}$, K.J. Anderson ${ }^{9}$, S. Anderson ${ }^{12}$, S. Arcelli ${ }^{17}$, S. Asai ${ }^{24}$, S.F. Ashby ${ }^{1}$, D. Axen ${ }^{29}$, G. Azuelos ${ }^{18, a}$, A.H. Ball ${ }^{17}$, E. Barberio ${ }^{8}$, R.J. Barlow ${ }^{16}$, J.R. Batley ${ }^{5}$, $\mathrm{S}_{\text {B Bamann }}^{3}$, J. Bechtluft ${ }^{14}$, T. Behnke ${ }^{27}$, K.W. Bell ${ }^{20}$, G. Bella ${ }^{23}$, A. Bellerive ${ }^{9}$, S. Bentvelsen ${ }^{8}$, S. Bethke $^{14}$, S. Betts $^{15}$, O. Biebel ${ }^{14}$, A. Biguzzi ${ }^{5}$, V. Blobel ${ }^{27}$, I.J. Bloodworth ${ }^{1}$, P. Bock ${ }^{11}$, J. Böhme ${ }^{14}$, D. Bonacorsi ${ }^{2}$, M. Boutemeur ${ }^{34}$, S. Braibant ${ }^{8}$, P. Bright-Thomas ${ }^{1}$, L. Brigliadori ${ }^{2}$, R.M. Brown ${ }^{20}$, H.J. Burckhart ${ }^{8}$, P. Capiluppi ${ }^{2}$, R.K. Carnegie ${ }^{6}$, A.A. Carter $^{13}$, J.R. Carter ${ }^{5}$, C.Y. Chang ${ }^{17}$, D.G. Charlton ${ }^{1, b}$, D. Chrisman ${ }^{4}$, C. Ciocca ${ }^{2}$, P.E.L. Clarke ${ }^{15}$, E. Clay ${ }^{15}$, I. Cohen ${ }^{23}$, J.E. Conboy ${ }^{15}$, O.C. Cooke ${ }^{8}$, C. Couyoumtzelis ${ }^{13}$, R.L. Coxe ${ }^{9}$, M. Cuffiani ${ }^{2}$, S. Dado ${ }^{22}$, G.M. Dallavalle ${ }^{2}$, R. Davis ${ }^{30}$, S. De Jong ${ }^{12}$, A. de Roeck ${ }^{8}$, P. Dervan ${ }^{15}$, K. Desch ${ }^{8}$, B. Dienes ${ }^{33, d}$, M.S. Dixit ${ }^{7}$, J. Dubbert ${ }^{34}$, E. Duchovni ${ }^{26}$, G. Duckeck ${ }^{34}$, I.P.Duerdoth ${ }^{16}$, P.G. Estabrooks ${ }^{6}$, E. Etzion ${ }^{23}$, F. Fabbri ${ }^{2}$, A. Fanfani ${ }^{2}$, M. Fanti ${ }^{2}$, A.A. Faust ${ }^{30}$, F. Fiedler ${ }^{27}$, M. Fierro ${ }^{2}$, I. Fleck ${ }^{10}$, R. Folman ${ }^{26}$, A. Frey ${ }^{8}$, A. Fürtjes ${ }^{8}$, D.I. Futyan ${ }^{16}$, P. Gagnon ${ }^{7}$, J.W. Gary ${ }^{4}$, J. Gascon ${ }^{18}$, S.M. Gascon-Shotkin ${ }^{17}$, G. Gaycken ${ }^{27}$, C. Geich-Gimbel ${ }^{3}$, G. Giacomelli ${ }^{2}$, P. Giacomelli ${ }^{2}$, V. Gibson ${ }^{5}$, W.R. Gibson ${ }^{13}$, D.M. Gingrich ${ }^{30, a}$, D. Glenzinski ${ }^{9}$, J. Goldberg ${ }^{22}$, W. Gorn ${ }^{4}$, C. Grandi ${ }^{2}$, K. Graham ${ }^{28}$, E. Gross ${ }^{26}$, J. Grunhaus $^{23}$, M. Gruwé27, G.G. Hanson ${ }^{12}$, M. Hansroul ${ }^{8}$, M. Hapke ${ }^{13}$, K. Harder ${ }^{27}$, A. Harel ${ }^{22}$, C.K. Hargrove ${ }^{7}$, M. Hauschild ${ }^{8}$, C.M. Hawkes ${ }^{1}$, R. Hawkings ${ }^{27}$, R.J. Hemingway ${ }^{6}$, M. Herndon ${ }^{17}$, G. Herten ${ }^{10}$, R.D. Heuer ${ }^{27}$, M.D. Hildreth ${ }^{8}$, J.C.Hill ${ }^{5}$, P.R. Hobson ${ }^{25}$, M. Hoch ${ }^{18}$, A. Hocker ${ }^{9}$, K. Hoffman ${ }^{8}$, R.J.Homer ${ }^{1}$, A.K. Honma ${ }^{28, a}$, D. Horváth ${ }^{32, c}$, K.R. Hossain ${ }^{30}$, R. Howard ${ }^{29}$, P. Hüntemeyer ${ }^{27}$, P. Igo-Kemenes ${ }^{11}$, D.C. Imrie ${ }^{25}$, K. Ishiii ${ }^{24}$, F.R. Jacob ${ }^{20}$, A. Jawahery ${ }^{17}$, H. Jeremie ${ }^{18}$, M. Jimack ${ }^{1}$, C.R. Jones ${ }^{5}$, P. Jovanovic ${ }^{1}$, T.R. Junk ${ }^{6}$, J. Kanzaki ${ }^{24}$, D. Karlen ${ }^{6}$, V. Kartvelishvili ${ }^{16}$, K. Kawagoe ${ }^{24}$, T. Kawamoto ${ }^{24}$, P.I. Kayal ${ }^{30}$, R.K. Keeler ${ }^{28}$, R.G. Kellogg ${ }^{17}$, B.W.Kennedy ${ }^{20}$, D.H. Kim ${ }^{19}$, A. Klier ${ }^{26}$, T. Kobayashi ${ }^{24}$, M. Kobel ${ }^{3, \text { e }}$, T.P. Kokott ${ }^{3}$, M. Kolrep ${ }^{10}$, S. Komamiya ${ }^{24}$, R.V. Kowalewski ${ }^{28}$, T. Kress ${ }^{4}$, P. Krieger ${ }^{6}$, J. von Krogh ${ }^{11}$, T. Kuhl ${ }^{3}$, P. Kyberd ${ }^{13}$, G.D. Lafferty ${ }^{16}$, H. Landsman ${ }^{22}$, D. Lanske ${ }^{14}$, J. Lauber ${ }^{15}$, S.R. Lautenschlager ${ }^{31}$, I. Lawson ${ }^{28}$, J.G. Layter ${ }^{4}$, A.M. Lee ${ }^{31}$, D. Lellouch ${ }^{26}$, J. Letts ${ }^{12}$, L. Levinson $^{26}$, R. Liebisch ${ }^{11}$, B. List ${ }^{8}$, C. Littlewood ${ }^{5}$, A.W. Lloyd ${ }^{1}$, S.L. Lloyd ${ }^{13}$, F.K. Loebinger ${ }^{16}$, G.D. Long ${ }^{28}$, M.J. Losty ${ }^{7}$, J. Lu ${ }^{29}$, J. Ludwig ${ }^{10}$, D. Liu ${ }^{12}$, A. Macchiolo ${ }^{2}$, A. Macpherson ${ }^{30}$, W. Mader ${ }^{3}$, M. Mannelli ${ }^{8}$, S. Marcellini $^{2}$, C. Markopoulos ${ }^{13}$, A.J. Martin ${ }^{13}$, J.P. Martin ${ }^{18}$, G. Martinez ${ }^{17}$, T. Mashimo ${ }^{24}$, P. Mättig ${ }^{26}$, W.J. McDonald ${ }^{30}$, J. McKenna $^{29}$, E.A. Mckigney ${ }^{15}$, T.J.McMahon ${ }^{1}$, R.A. McPherson ${ }^{28}$, F. Meijers ${ }^{8}$, S. Menke ${ }^{3}$, F.S. Merritt ${ }^{9}$, H. Mes ${ }^{7}$, J. Meyer $^{27}$, A. Michelini ${ }^{2}$, S. Mihara ${ }^{24}$, G. Mikenberg ${ }^{26}$, D.J. Miller ${ }^{15}$, R. Mir ${ }^{26}$, W. Mohr ${ }^{10}$, A. Montanari $^{2}$, T. Mori ${ }^{24}$, K. Nagai ${ }^{8}$, I. Nakamura ${ }^{24}$, H.A. Neal ${ }^{12}$, R. Nisius ${ }^{8}$, S.W. O’Neale ${ }^{1}$, F.G. Oakham ${ }^{7}$, F. Odorici ${ }^{2}$, H.O. Ogren ${ }^{12}$, M.J. Oreglia ${ }^{9}$, S. Orito ${ }^{24}$, J.Pálinkás ${ }^{33, d}$, G.Pásztor ${ }^{32}$, J.R. Pater ${ }^{16}$, G.N.Patrick ${ }^{20}$, J.Patt ${ }^{10}$, R. Perez-Ochoa ${ }^{8}$, S. Petzold ${ }^{27}$, P. Pfeifenschneider ${ }^{14}$, J.E. Pilcher ${ }^{9}$, J. Pinfold ${ }^{30}$, D.E. Plane ${ }^{8}$, P. Poffenberger ${ }^{28}$, B. Poli ${ }^{2}$, J. Polok ${ }^{8}$, M. Przybycień ${ }^{8, f}$, C. Rembser ${ }^{8}$, H. Rick ${ }^{8}$, S. Robertson ${ }^{28}$, S.A. Robins ${ }^{22}$, N. Rodning ${ }^{30}$, J.M. Roney ${ }^{28}$, S. Rosati ${ }^{3}$, K. Roscoe $^{16}$, A.M. Rossi ${ }^{2}$, Y. Rozen ${ }^{22}$, K. Runge ${ }^{10}$, O. Runolfsson ${ }^{8}$, D.R. Rust ${ }^{12}$, K. Sachs ${ }^{10}$, T. Saeki ${ }^{24}$, O. Sahr ${ }^{34}$, W.M. Sang ${ }^{25}$, E.K.G. Sarkisyan ${ }^{23}$, C. Sbarra ${ }^{29}$, A.D. Schaile ${ }^{34}$, O. Schaile ${ }^{34}$, P. Scharff-Hansen ${ }^{8}$, J. Schieck ${ }^{11}$, S. Schmitt ${ }^{11}$, A. Schöning ${ }^{8}$, M. Schröder ${ }^{8}$, M. Schumacher ${ }^{3}$, C. Schwick ${ }^{8}$, W.G. Scott ${ }^{20}$, R. Seuster ${ }^{14}$, T.G. Shears ${ }^{8}$, B.C. Shen ${ }^{4}$, C.H. Shepherd-Themistocleous ${ }^{8}$, P. Sherwood ${ }^{15}$, G.P.Siroli ${ }^{2}$, A.Sittler ${ }^{27}$, A.Skuja ${ }^{17}$, A.M. Smith ${ }^{8}$, G.A.Snow ${ }^{17}$, R. Sobie ${ }^{28}$, S. Söldner-Rembold ${ }^{10}$, S. Spagnolo ${ }^{20}$, M.Sproston ${ }^{20}$, A.Stahl ${ }^{3}$, K. Stephens ${ }^{16}$, J. Steuerer ${ }^{27}$, K. Stoll ${ }^{10}$, D. Strom ${ }^{19}$, R. Ströhmer ${ }^{34}$, B. Surrow ${ }^{8}$, S.D. Talbot ${ }^{1}$, P. Taras ${ }^{18}$, S. Tarem ${ }^{22}$, R. Teuscher ${ }^{8}$, M. Thiergen ${ }^{10}$, J. Thomas ${ }^{15}$, M.A. Thomson ${ }^{8}$, E. Torrence ${ }^{8}$, S. Towers ${ }^{6}$, I. Trigger ${ }^{18}$, Z. Trócsányi ${ }^{33}$, E. Tsur ${ }^{23}$, A.S. Turcot ${ }^{9}$, M.F. Turner-Watson ${ }^{1}$, I. Ueda ${ }^{24}$, R. Van Kooten ${ }^{12}$, P. Vannerem ${ }^{10}$, M. Verzocchi ${ }^{10}$, H. Voss ${ }^{3}$, F. Wäckerle ${ }^{10}$, A. Wagner ${ }^{27}$, C.P. Ward ${ }^{5}$, D.R. Ward ${ }^{5}$, P.M. Watkins ${ }^{1}$, A.T. Watson ${ }^{1}$, N.K. Watson ${ }^{1}$, P.S. Wells ${ }^{8}$, N. Wermes ${ }^{3}$, J.S. White ${ }^{6}$, G.W.Wilson ${ }^{16}$, J.A. Wilson ${ }^{1}$, T.R. Wyatt ${ }^{16}$, S. Yamashita ${ }^{24}$, G. Yekutieli ${ }^{26}$, V.Zacek ${ }^{18}$, D. Zer-Zion ${ }^{8}$

${ }^{1}$ School of Physics and Astronomy, University of Birmingham, Birmingham B15 2TT, UK

2 Dipartimento di Fisica dell' Università di Bologna and INFN, I-40126 Bologna, Italy

${ }^{3}$ Physikalisches Institut, Universität Bonn, D-53115 Bonn, Germany

${ }^{4}$ Department of Physics, University of California, Riverside CA 92521, USA

${ }^{5}$ Cavendish Laboratory, Cambridge CB3 0HE, UK

${ }^{6}$ Ottawa-Carleton Institute for Physics, Department of Physics, Carleton University, Ottawa, Ontario K1S 5B6, Canada

${ }^{7}$ Centre for Research in Particle Physics, Carleton University, Ottawa, Ontario K1S 5B6, Canada 
${ }^{8}$ CERN, European Organisation for Particle Physics, CH-1211 Geneva 23, Switzerland

${ }^{9}$ Enrico Fermi Institute and Department of Physics, University of Chicago, Chicago IL 60637, USA

${ }^{10}$ Fakultät für Physik, Albert Ludwigs Universität, D-79104 Freiburg, Germany

${ }^{11}$ Physikalisches Institut, Universität Heidelberg, D-69120 Heidelberg, Germany

12 Indiana University, Department of Physics, Swain Hall West 117, Bloomington IN 47405, USA

13 Queen Mary and Westfield College, University of London, London E1 4NS, UK

14 Technische Hochschule Aachen, III Physikalisches Institut, Sommerfeldstrasse 26-28, D-52056 Aachen, Germany

${ }^{15}$ University College London, London WC1E 6BT, UK

${ }^{16}$ Department of Physics, Schuster Laboratory, The University, Manchester M13 9PL, UK

17 Department of Physics, University of Maryland, College Park, MD 20742, USA

18 Laboratoire de Physique Nucléaire, Université de Montréal, Montréal, Quebec H3C 3J7, Canada

19 University of Oregon, Department of Physics, Eugene OR 97403, USA

${ }^{20}$ CLRC Rutherford Appleton Laboratory, Chilton, Didcot, Oxfordshire OX11 0QX, UK

22 Department of Physics, Technion-Israel Institute of Technology, Haifa 32000, Israel

${ }^{23}$ Department of Physics and Astronomy, Tel Aviv University, Tel Aviv 69978, Israel

${ }^{24}$ International Centre for Elementary Particle Physics and Department of Physics, University of Tokyo, Tokyo 113-0033, and

Kobe University, Kobe 657-8501, Japan

${ }^{25}$ Institute of Physical and Environmental Sciences, Brunel University, Uxbridge, Middlesex UB8 3PH, UK

${ }^{26}$ Particle Physics Department, Weizmann Institute of Science, Rehovot 76100, Israel

27 Universität Hamburg/DESY, II Institut für Experimental Physik, Notkestrasse 85, D-22607 Hamburg, Germany

${ }^{28}$ University of Victoria, Department of Physics, P O Box 3055, Victoria BC V8W 3P6, Canada

${ }^{29}$ University of British Columbia, Department of Physics, Vancouver BC V6T 1Z1, Canada

${ }^{30}$ University of Alberta, Department of Physics, Edmonton AB T6G 2J1, Canada

31 Duke University, Dept of Physics, Durham, NC 27708-0305, USA

32 Research Institute for Particle and Nuclear Physics, H-1525 Budapest, P O Box 49, Hungary

${ }^{33}$ Institute of Nuclear Research, H-4001 Debrecen, P O Box 51, Hungary

${ }^{34}$ Ludwigs-Maximilians-Universität München, Sektion Physik, Am Coulombwall 1, D-85748 Garching, Germany

Received: 24 February 1999 / Published online: 8 September 1999

Abstract. Gluon jets are identified in hadronic $\mathrm{Z}^{0}$ decays as all the particles in a hemisphere opposite to a hemisphere containing two tagged quark jets. Gluon jets defined in this manner are equivalent to gluon jets produced from a color singlet point source and thus correspond to the definition employed for most theoretical calculations. In a separate stage of the analysis, we select quark jets in a manner to correspond to calculations, as the particles in hemispheres of flavor tagged light quark (uds) events. We present the distributions of rapidity, scaled energy, the logarithm of the momentum, and transverse momentum with respect to the jet axes, for charged particles in these gluon and quark jets. We also examine the charged particle multiplicity distributions of the jets in restricted intervals of rapidity. For soft particles at large $p_{\mathrm{T}}$, we observe the charged particle multiplicity ratio of gluon to quark jets to be $2.29 \pm 0.09$ (stat.) \pm 0.15 (syst.), in agreement with the prediction that this ratio should approximately equal the ratio of QCD color factors, $\mathrm{C}_{\mathrm{A}} / \mathrm{C}_{\mathrm{F}}=2.25$. The intervals used to define soft particles and large $p_{\mathrm{T}}$ for this result, $p<4 \mathrm{GeV} / c$ and $0.8<p_{\mathrm{T}}<3.0 \mathrm{GeV} / c$, are motivated by the predictions of the Herwig Monte Carlo multihadronic event generator. Additionally, our gluon jet data allow a sensitive test of the phenomenon of non-leading QCD terms known as color reconnection. We test the model of color reconnection implemented in the Ariadne Monte Carlo multihadronic event generator and find it to be disfavored by our data. 


\section{Introduction}

Gluon jets have been a subject of intensive experimental investigation since the time of their first observation [1]. It has proven difficult to obtain theoretically meaningful information about the internal properties of gluon jets, however, due to the experimental difficulty of identifying

${ }^{\mathrm{a}}$ and at TRIUMF, Vancouver, Canada V6T 2A3

$\mathrm{b}$ and Royal Society University Research Fellow

c and Institute of Nuclear Research, Debrecen, Hungary

d and Department of Experimental Physics, Lajos Kossuth University, Debrecen, Hungary

${ }^{\mathrm{e}}$ on leave of absence from the University of Freiburg

${ }^{\mathrm{f}}$ and University of Mining and Metallurgy, Cracow gluon jets in a manner which corresponds to calculations in Quantum Chromodynamics (QCD). The theoretical description of gluon jets usually relies on the creation of a gluon jet pair, gg, from a color singlet point source, allowing an unambiguous definition of the gluon jet's properties by summing inclusively over the particles in an event hemisphere. Point-source creation of a high energy gg pair $\left(E_{\text {jet }}>5 \mathrm{GeV}\right)$ is not a process which has been observed in nature, however. ${ }^{1}$ Instead, experimenters have relied on jet finding algorithms to isolate high energy gluon jets within other types of events. At $\mathrm{e}^{+} \mathrm{e}^{-}$colliders, most stud-

\footnotetext{
${ }^{1}$ It is possible to identify a pure source of gg events in radiative $\Upsilon$ decays [2]; however, the jet energies are only about $5 \mathrm{GeV}$ in this case, which limits their usefulness for jet studies
} 
ies of gluon jets employ a jet finding algorithm to select a sample of three-jet $q \bar{q} g$ events. The same jet finder is used to divide the particles of an event into a gluon jet part and two quark jet parts. At hadron colliders, jet finding algorithms are used to select two-jet gg events which do not arise from a point source since the gluon jets are color-connected to other jets and to the underlying event from the proton remnants. Jet finders are used to artificially divide events into gluon and non-gluon jet parts similarly to the $\mathrm{e}^{+} \mathrm{e}^{-}$case. The results obtained for the gluon jet properties at either type of collider are found to depend strongly on the jet finding algorithm and, as a consequence, have limited theoretical significance.

In [3], a method was introduced to experimentally identify gluon jets in a manner which yields a close correspondence to the theoretical definition. The method is based on rare events of the type $\mathrm{e}^{+} \mathrm{e}^{-} \rightarrow \mathrm{q} \overline{\mathrm{q}} \mathrm{g}_{\text {incl. }}$, in which the $\mathrm{q}$ and $\bar{q}$ are identified quark jets ${ }^{2}$ which appear in the same hemisphere of an $\mathrm{e}^{+} \mathrm{e}^{-}$multihadronic annihilation event. The object $\mathrm{g}_{\text {incl. }}$, taken to be the gluon jet, is defined by all particles observed in the hemisphere opposite to that containing the $\mathrm{q}$ and $\overline{\mathrm{q}}$. The properties of gluon jets found using this method are almost entirely independent of the choice of the jet finding algorithm used to define the quark jets. In the limit that the $\mathrm{q}$ and $\overline{\mathrm{q}}$ are collinear, the gluon

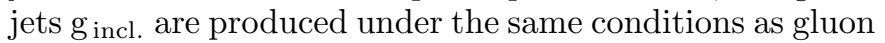
jets in gg events [4]. The $\mathrm{g}_{\text {incl. }}$ jets therefore correspond closely to single gluon jets in gg events, defined by dividing the gg events in half using the plane perpendicular to the principal event axis.

In several previous studies $[5,6]$, we employed the $\mathrm{g}_{\text {incl. }}$ hemisphere method of defining gluon jets to determine the charged particle multiplicity distribution of the jets. The data were collected using the OPAL detector at the $\mathrm{e}^{+} \mathrm{e}^{-}$collider LEP at CERN. In this paper, we extend our investigation of gluon jets to other distributions, in particular to rapidity, the logarithm of momentum, transverse momentum with respect to the jet axis, scaled energy, and multiplicity in restricted rapidity intervals, for charged particles in the jets. The results for gluon jets are compared to those of light flavored (uds) quark jets. We define a uds jet to be all the particles in a hemisphere of an $\mathrm{e}^{+} \mathrm{e}^{-} \rightarrow \mathrm{Z}^{0} \rightarrow$ hadrons event in which the $\mathrm{Z}^{0}$ decays into a quark pair $q \overline{\mathrm{q}}$ with $\mathrm{q}=\mathrm{u}$, $\mathrm{d}$ or $\mathrm{s}$. Use of light quark events results in a better correspondence between the data and the massless quark assumption employed for most theoretical calculations. Use of event hemispheres to define the quark jets yields an inclusive definition analogous to that of the gluon jets.

Figure 1 illustrates the three types of event pertinent to our study. Figure 1a shows a diagram for gg production from a color singlet point source. The production of $\mathrm{g}_{\text {incl. }}$ jets in $\mathrm{e}^{+} \mathrm{e}^{-}$annihilations, providing an experimentally accessible source of high energy gluon jets with nearly identical properties to the gluon jets in gg events, is shown in Fig. 1b. Last, Fig. 1c shows uds jet production in $\mathrm{e}^{+} \mathrm{e}^{-}$ annihilations.

\footnotetext{
${ }^{2}$ In this analysis we make no distinction between quark and antiquark jets and refer to both as "quark" jets
}

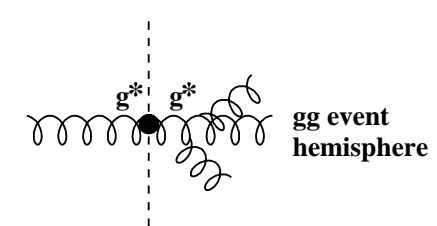

(a)

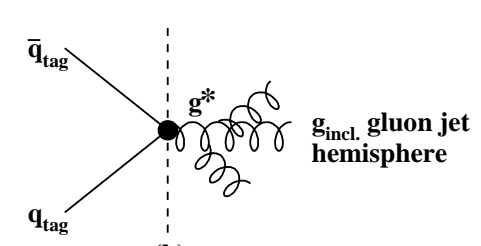

(b)

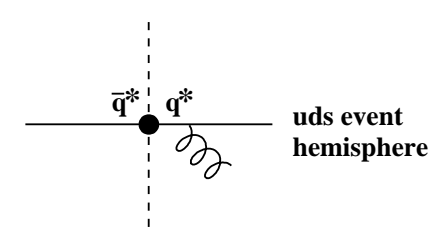

(c)

Fig. 1a-c. Event types pertinent to this analysis. The dashed vertical lines represent hemisphere boundaries, defined in our study by the plane perpendicular to the thrust axis, while the large solid dots represent a color singlet point source. a gg production. $\mathbf{b} \mathrm{e}^{+} \mathrm{e}^{-} \rightarrow \mathrm{q}_{\text {tag }} \overline{\mathrm{q}}_{\text {tag }} \mathrm{g}_{\text {incl. }}$ : The quark jets $\mathrm{q}_{\text {tag }}$ and $\bar{q}_{\text {tag }}$ are tagged $b$ jets defined using a jet algorithm and are used only as a tool to identify the $\mathrm{g}_{\text {incl. }}$ jet hemispheres. The $\mathrm{g}_{\text {incl. }}$ jet hemispheres provide the gluon jet sample for our study. The $\mathrm{g}_{\text {incl. }}$ jets yield virtually the same results for the experimental observables in our study as the hemispheres of gg events shown in part a. $\mathbf{c} \mathrm{e}^{+} \mathrm{e}^{-} \rightarrow \mathrm{q} \overline{\mathrm{q}}$, with q a light (uds) quark: Hemispheres in these events provide the quark jet sample for our study

A topic of recent interest is that of color reconnection [7]. The phenomenon of color reconnection expresses the possibility that certain non-leading terms usually ignored in QCD calculations can drastically influence the color singlet structure of an event. Most recent attention to color reconnection has focused on its implications for the $\mathrm{W}$ boson mass measurement at LEP-2 [8]. Color reconnection is an interesting phenomenon in its own right, however, as a basic issue of QCD interference and confinement. In $\mathrm{e}^{+} \mathrm{e}^{-} \rightarrow \mathrm{Z}^{0} \rightarrow$ hadrons events, color reconnection is expected to occasionally yield an event in which a pure system of gluons hadronizes in isolation from the rest of the event (see $[8,9]$ and the discussion below in Sect. 11). Such events are expected to markedly affect the mean properties of events in which the initial quark and antiquark from the decay of the $\mathrm{Z}^{0}$ recoil against a gluon jet, as in our selected $\mathrm{e}^{+} \mathrm{e}^{-} \rightarrow \mathrm{q} \overline{\mathrm{q}} \mathrm{g}_{\text {incl. }}$ sample. Thus, our gluon jet data can provide a sensitive test of the color reconnection phenomenon. In this paper, we use our $\mathrm{g}_{\text {incl. data to }}$ perform the most stringent test to date of the model for color reconnection [10] implemented in the Ariadne Monte Carlo multihadronic event generator [11], version 4.08 . 


\section{Detector and data sample}

The OPAL detector is described in detail elsewhere [12, 13]. The tracking system consists of a silicon microvertex detector, an inner vertex chamber, a large volume jet chamber and specialized chambers at the outer radius of the jet chamber which improve the measurements in the $z$-direction. ${ }^{3}$ The tracking system covers the region $|\cos \theta|<0.98$ and is enclosed by a solenoidal magnet coil with an axial field of $0.435 \mathrm{~T}$. Electromagnetic energy is measured by a lead-glass calorimeter located outside the magnet coil, which also covers $|\cos \theta|<0.98$.

The present analysis is based on a sample of about 3708000 hadronic $\mathrm{Z}^{0}$ decay events, corresponding to our data sample from LEP-1 which includes readout of the silicon strip microvertex detector [13]: 998940 of these events were collected in 1991 and 1992 when our microvertex detector was instrumented for readout of the $r-\phi$ coordinate only, while the remainder of the events, collected from 1993 to 1995 , contain readout of both the $r-\phi$ and $z$ coordinates. The procedures for identifying hadronic events are discussed in [14]. Charged tracks and electromagnetic clusters were selected for the analysis as follows. Charged tracks were required to have at least 20 measured points (of 159 possible) in the jet chamber, to have a momentum greater than $0.10 \mathrm{GeV} / c$, to lie in the region $|\cos \theta|<0.94$, and to point to the origin to within $5 \mathrm{~cm}$ in the $r$ - $\phi$ plane. In addition, they were required to yield a $\chi^{2}$ per degreeof-freedom of less than 100 for the track fit in the $r$ - $\phi$ plane. Clusters were required to be spread over at least two lead glass blocks and to have an energy greater than $0.10 \mathrm{GeV}$ if they were in the barrel section of the detector $(|\cos \theta|<0.82)$ or greater than $0.30 \mathrm{GeV}$ if they were in the endcap section $(0.82<|\cos \theta|<0.98)$. Each accepted track and cluster was considered to be a particle. Tracks were assigned the pion mass. Clusters were assigned zero mass since they originate mostly from photons. To eliminate residual background and events in which a significant number of particles was lost near the beam direction, the number of accepted charged tracks in each event was required to be at least five and the thrust axis [15] of the event, calculated using the particles, was required to satisfy $\left|\cos \left(\theta_{\text {thrust }}\right)\right|<0.90$, where $\theta_{\text {thrust }}$ is the angle between the thrust and beam axes. The residual background to the sample of hadronic events from all sources was estimated to be less than $1 \%$.

\section{Gluon jet selection}

For this study, a gluon jet is defined inclusively as the particles in an $\mathrm{e}^{+} \mathrm{e}^{-}$event hemisphere opposite to a hemisphere containing two identified quark jets, as stated in the introduction. To select the $g_{\text {incl. }}$ gluon jets, each event is divided into hemispheres using the plane perpendicular to

\footnotetext{
3 Our coordinate system is defined so that $z$ is the coordinate parallel to the $\mathrm{e}^{-}$beam axis, $r$ is the coordinate normal to the beam axis, $\phi$ is the azimuthal angle around the beam axis and $\theta$ is the polar angle with respect to $z$
}

the thrust axis. The procedures described below are applied to each hemisphere separately. For the purpose of identifying two quark jets in a single hemisphere, we employ the $k_{\perp}$ ("Durham") jet algorithm [16]. The results for the gluon jet properties are almost entirely insensitive to this choice of jet algorithm, as is discussed in [3] (see also Sect. 8). Note that a jet algorithm is used only as a selection tool for the $g_{\text {incl. }}$ jets, not for the analysis of quark jet properties. The manner in which quark jets are selected so as to correspond to the definition employed by analytic calculations is presented in Sect. 4. The resolution parameter of the jet algorithm is adjusted to yield exactly two reconstructed jets in a hemisphere. Next, we attempt to reconstruct a displaced secondary vertex in each of the two jets. Displaced secondary vertices are associated with heavy quark decay, especially that of the b quark. At LEP, b quarks are produced almost exclusively at the electroweak vertex ${ }^{4}$ : thus a jet containing a $\mathrm{b}$ hadron is almost always a quark jet.

To reconstruct secondary vertices in jets, we employ the method described in [19]. Briefly, charged tracks are selected for the secondary vertex reconstruction procedure if they have coordinate information from at least one of the two silicon detector layers, if their momentum is larger than $0.5 \mathrm{GeV} / c$, and if their distance of closest approach to the primary event vertex [19] is less than $0.3 \mathrm{~cm}$. Additionally, we require the maximum uncertainty on the distance of closest approach to be $0.1 \mathrm{~cm}$. For the 1991-92 data (with only $r-\phi$ coordinate readout of the microvertex detector), the distance of closest approach, and the distances $b$ and $L$ discussed below, are determined in the $r$ - $\phi$ plane. For the 1993-95 data (with $r$ - $\phi$ and $z$ coordinate readout of the microvertex detector), these distances are determined in three dimensions. A secondary vertex is required to contain at least three tracks which satisfy the above criteria. For the 1991-92 data, at least two of these tracks are required to satisfy $b / \sigma_{b}>2.5$, where $b$ is the signed impact parameter value of a track with respect to the primary event vertex and $\sigma_{b}$ is the uncertainty associated with $b$. For the 1993-95 data, only one track in the secondary vertex is required to have $b / \sigma_{b}>2.5$. For jets with such a secondary vertex, the signed decay length, $L$, is calculated with respect to the primary vertex, along with its error, $\sigma_{L}$. The sign of $L$ is determined by summing the three momenta of the tracks fitted to the secondary vertex; $L>0$ if the secondary vertex is displaced from the primary vertex in the same hemisphere as this momentum sum, and $L<0$ otherwise. The sign of $b$ is determined in an analogous manner. More details concerning the determination of $L$ and $b$ are given in [19]. To be tagged as a quark jet, a jet is required to have a visible energy of at least $10 \mathrm{GeV}$ and a successfully reconstructed secondary vertex with $L / \sigma_{L}>3.5$ for the 1991-92 data or $L / \sigma_{L}>5.0$ for the 1993-95 data. The visible energy of a jet is defined by the sum of the energies of the particles assigned to the

\footnotetext{
${ }^{4}$ About $22 \%$ of hadronic $\mathrm{Z}^{0}$ events contain a $\mathrm{b} \overline{\mathrm{b}}$ quark pair from the electroweak decay of the $\mathrm{Z}^{0}[17]$ compared to only about $0.2 \%$ with a b $\bar{b}$ pair from gluon splitting [18]
} 
jet. We refer to a hemisphere with two tagged jets as a tagged hemisphere.

We next examine the angles that the two jets in a tagged hemisphere make with respect to the thrust axis and to each other. If the two jets are close together, or if one of the two jets is much more energetic than the other, it is very likely that one of the two jets is a gluon jet due to the strong kinematic similarity to an event with gluon radiation from a quark. To reduce this background, we require the angle between each jet and the thrust axis to exceed $15^{\circ}$ and the angle between the two jets to exceed $70^{\circ}$. We further require the two jets to lie no more than $70^{\circ}$ from the thrust axis to eliminate jets near the hemisphere boundary. These angular restrictions on the quark jet directions do not affect the good correspondence between $\mathrm{g}_{\text {incl. }}$ jets from $\mathrm{e}^{+} \mathrm{e}^{-}$annihilations and hemispheres of $\mathrm{gg}$ events from a point source, as is demonstrated below in Sect. 6. Last, we eliminate events with three tagged jets, i.e. events in which both jets in one hemisphere and one of the two jets in the other hemisphere have been tagged as b jets (about $4 \%$ of the events after the other cuts have been applied), because Monte Carlo study shows them to be mostly background. ${ }^{5}$ There are no events in which both jets in both hemispheres are tagged. In total, 439 events

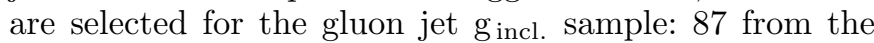
1991-92 data and 352 from the 1993-95 data. The mean angle between the two tagged quark jets in the final $\mathrm{g}_{\text {incl. }}$ jet sample is $91.5^{\circ}$ with a standard deviation of $12.8^{\circ}$.

The purity of this sample is estimated using the Jetset Monte Carlo multihadronic event generator [20] including detector simulation [21] and the same analysis procedures as are applied to the data. For the simulation of the 1991-92 data, we use a combination of events generated using version 7.3 of the program with the parameter values given in [22] and of events generated using version 7.4 of the program with the parameter values given in [23]. The initial Monte Carlo samples have about 3000000 events for version 7.3 and 1000000 events for version 7.4. The two Jetset versions yield results which are consistent with each other to within the statistical uncertainties and so we combine them. For the simulation of the 1993-95 data, we use a sample of about 6000000 events generated using version 7.4 with the parameters given in [23]. The hadron level Monte Carlo jets are examined to determine whether they are associated with an underlying quark or antiquark jet. To perform this association, the Monte Carlo events are also examined at the parton level. We determine the directions of the primary quark and antiquark from the $\mathrm{Z}^{0}$ decay after the parton shower has terminated. Of the four hadron level jets reconstructed in an event (two in each hemisphere), the jet closest to the direction of an evolved primary quark or antiquark is considered to be a quark jet. The distinct hadron jet closest

${ }^{5}$ The Monte Carlo predicts that about $40 \%$ of these events arise from gluon splitting to $\mathrm{a} b \overline{\mathrm{b}}$ or $c \overline{\mathrm{c}}$ pair; although rare in inclusive $\mathrm{Z}^{0}$ decays, our analysis preferentially selects such events. The other events with three tagged jets have a mistagged gluon jet due to the presence of a long lived particle such as a $\Lambda$ baryon to the evolved primary quark or antiquark not associated with this first hadron jet is considered to be the other quark jet. The primary quark and antiquark are closest to different hadron jets in $99.5 \%$ of the selected events. The mean angle between the primary quark or antiquark and its associated hadron jet is $3.9^{\circ}$ in these events. An event in which one of the two jets in the tagged hemisphere is not identified as a quark jet is deemed to be a background event. Using this algorithm, we estimate the purity of the $g_{\text {incl. }}$ sample to be $(78.8 \pm 2.4$ (stat.) $) \%$ for the 1991-92 data and ( $82.9 \pm 1.4$ (stat.))\% for the 1993-95 data. The estimated purity of the combined 1991-1995 sample is $(81.9 \pm 1.2$ (stat.))\%. The background events mostly arise when two tracks from a long lived particle such as a $\mathrm{K}_{\mathrm{S}}^{0}$ or $\Lambda$ are combined with a third track to define a secondary vertex in a gluon jet, or else from events in which a gluon decays into a b $\bar{b}$ pair. About $94 \%$ of the events in the $\mathrm{g}_{\text {incl. }}$ sample are predicted to be $\mathrm{b}$ events. This reliance on $b$ events is not expected to affect our results since the properties of hard, acollinear gluon jets do not depend on the event flavor according to QCD. More details are given in $[5]$.

The $g_{\text {incl. }}$ tag rates, defined by the ratio of the number of $\mathrm{g}_{\text {incl. }}$ jets to the number of events in the initial inclusive multihadronic event samples, are $(8.71 \pm 0.94$ (stat. $)) \times$ $10^{-5}$ for the data and $(7.20 \pm 0.42$ (stat. $\left.)\right) \times 10^{-5}$ for the Monte Carlo for the 1991-92 analysis, and $(1.30 \pm 0.07$ (stat.) $) \times 10^{-4}$ for the data and $(1.25 \pm 0.05$ (stat. $\left.)\right) \times 10^{-4}$ for the Monte Carlo for the 1993-95 analysis. Thus the Monte Carlo reproduces the measured tag rates well. The tag rate for the 1993-95 data is substantially larger than that for the 1991-92 data as a consequence of the addition of $z$ coordinate readout from the silicon microvertex detector.

The energy of the $g_{\text {incl. }}$ jet is determined by imposing overall energy-momentum conservation on the system of three jets comprised of the $\mathrm{g}_{\text {incl. }}$ jet and the two jets in the tagged hemisphere. A direction is determined for the $\mathrm{g}_{\text {incl. }}$ jet by summing the momenta of the particles in its hemisphere. The jet directions are used in conjunction with the jet velocities to calculate the jet energies, assuming massive kinematics. ${ }^{6}$ We obtain $\langle E\rangle_{\mathrm{g}_{\text {incl. }}}=40.1 \pm$ 0.2 (stat.) GeV. This value includes a multiplicative correction of 1.03 to account for the effects of detector response and initial-state photon radiation. The correction factor is obtained using Monte Carlo predictions with and without simulation of the detector as is described in [5] (see also Sect. 7). The correction procedure accounts for any possible double counting of particle energy in the determination of the jet's visible energy, which could arise since we use both charged track and electromagnetic clus-

\footnotetext{
${ }^{6}$ For a system of three jets labelled 1, 2 and 3, the energymomentum constraints $\Sigma_{i=1}^{3} E_{\text {jet } i=E_{\text {c.m. }} \text { and } \Sigma_{i=1}^{3} \vec{P}_{\text {jet } i}=0}$ are solved for $E_{\text {jet } i}$, where $\vec{P}_{\text {jet } i}=\vec{\beta}_{i} E_{\text {jet } i}$ is the momentum of jet $i$, with its velocity $\vec{\beta}_{i}$ given by its visible 3 momentum divided by its visible energy. The solution is $E_{\text {jet } i}=$ $E_{\text {c.m. }} \beta_{j} \beta_{k} \sin \theta_{i} /\left(\beta_{1} \beta_{2} \sin \theta_{3}+\beta_{1} \beta_{3} \sin \theta_{2}+\beta_{2} \beta_{3} \sin \theta_{1}\right)$ where $\theta_{1}, \theta_{2}$ and $\theta_{3}$ are the angles between the jets with $\theta_{i}$ opposite to jet $i$, and where $(i, j, k)=(1,2,3),(2,3,1)$ or $(3,1,2)$
} 
ter information. The corrected mean visible energy of the $\mathrm{g}_{\text {incl. }}$ jets is $40.8 \pm 0.4$ (stat.) GeV. The difference between the mean calculated and visible jet energies is used to define a systematic uncertainty. The mean energy of the gluon jets in our study is therefore $\langle E\rangle_{\mathrm{g}_{\text {incl. }}}=40.1 \pm$ 0.2 (stat.) \pm 0.7 (syst.) $\mathrm{GeV}$.

\section{Light quark jet selection}

To select quark jets in a manner which corresponds to analytic calculations, we define quark jets inclusively as the particles in hemispheres of light (uds) flavored $\mathrm{e}^{+} \mathrm{e}^{-} \rightarrow$ $\mathrm{Z}^{0} \rightarrow$ hadrons events. Note that these are not the same as the quark jets discussed in the previous section (defined using the $k_{\perp}$ jet algorithm), which are used only as a tool to identfy $\mathrm{g}_{\text {incl. }}$ gluon jets. Due to the relatively large efficiency of the uds jet selection procedure (see below), it is not necessary to employ the entire sample of about 3708000 events mentioned in Sect. 2 for the uds jet analysis. Instead, we base this analysis on an initial sample of 222921 hadronic annihilation events with c.m. energies within $100 \mathrm{MeV}$ of the $\mathrm{Z}^{0}$ peak. In addition to the selection criteria described in Sect. 2, we require the angle $\theta_{\text {thrust }}$ between the thrust and beam axes to satisfy $\left|\cos \left(\theta_{\text {thrust }}\right)\right|<0.70$ for this analysis, to contain the events well within the geometric acceptance of the silicon microvertex detector.

The uds jet tagging is based on the signed impact parameter values of charged tracks with respect to the primary event vertex, $b$, since the distribution of this variable is strongly skewed toward positive values for $\mathrm{c}$ and $\mathrm{b}$ events but not for uds events. Charged tracks are selected for the uds tagging procedure if they have $r-\phi$ coordinate information from at least one silicon detector layer, a momentum of $0.5 \mathrm{GeV} / c$ or larger, and a maximum distance of closest approach to the primary event vertex in the $r-\phi$ plane of $0.3 \mathrm{~cm}$ with a maximum uncertainty on this quantity of $0.1 \mathrm{~cm}$. If no track in an event satisfies these requirements $(0.003 \%$ of the events), the event is eliminated. The number of tracks which meet these requirements and which have $b / \sigma_{b}>2.5$ in the $r$ - $\phi$ plane is determined. An event is tagged as containing a uds jet if this number is zero. In total, 53552 events are tagged. Both hemispheres of a tagged event are identified as uds jet hemispheres and are used in the subsequent analysis: thus, there are 107104 uds jets in our study. The estimated uds purity of this sample, obtained by treating Jetset events with detector simulation in the same manner as the data, is $(86.4 \pm 0.3$ (stat.) $) \%$. The Monte Carlo predicts that $86 \%$ of the background events are c events and that $14 \%$ are b events. The uds jet tag rate, defined by the ratio of the number of identified uds jets to the number of events in the initial inclusive multihadronic event sample, is $0.480 \pm 0.002$ (stat.) for the data and $0.487 \pm 0.001$ (stat.) for the Monte Carlo: thus the measured and simulated tag rates agree to better than $1 \%$. The energy of the uds jets is given by the beam energy, $45.6 \mathrm{GeV}$, with essentially no uncertainty.

\section{Experimental distributions}

In this study, we examine the distributions of rapidity, scaled energy, the logarithm of the momentum, transverse momentum with respect to the jet axis, and multiplicity in restricted rapidity intervals, of charged particles in the $\mathrm{g}_{\text {incl. }}$ and uds jets. All these variables are commonly used to characterize the energy and multiplicity structure of jets.

Rapidity, $y$, is defined by

$$
y=\frac{1}{2} \ln \left(\frac{E+\vec{p} \cdot \hat{r}}{E-\vec{p} \cdot \hat{r}}\right)
$$

with $E$ and $\vec{p}$ the energy and momentum of a particle and $\hat{r}$ the axis with respect to which rapidity is calculated. We choose $\hat{r}$ to be the sphericity axis [24] calculated using the charged and neutral particles in the $\mathrm{g}_{\text {incl. }}$ or uds jets. We do not use the thrust axis to calculate rapidity, contrary to common usage, because the thrust axis is used to determine the hemisphere boundaries of the $g_{\text {incl. }}$ and uds jets and we wish to reduce the correlation between the event selection and the jet analysis. ${ }^{7}$

The scaled energy of a particle, $x_{E}$, is given by

$$
x_{E}=\frac{E}{E_{\text {jet }}}
$$

with $E_{\text {jet }}$ determined as explained in Sects. 3 and 4 . The distribution of $x_{E}$ is commonly referred to as the fragmentation function.

We also study the distributions of $\ln (p)$, with $p$ the particle momentum, and of $p_{\mathrm{T}}$, the transverse momentum of particles with respect to the jet axis, as stated above. The jet axis for the $p_{\mathrm{T}}$ calculation is defined by summing the momenta of the particles in the $g_{\text {incl. }}$ or uds jets. Besides the inclusive $p_{\mathrm{T}}$ distribution, we examine the $p_{\mathrm{T}}$ distribution of soft particles, defined as particles with momenta below $4 \mathrm{GeV} / c$ : we refer to this distribution as $p_{\mathrm{T}}(p<4 \mathrm{GeV} / c)$. The motivation for including this last variable in our study is presented in Sect. 9.2.

Last, we study the distribution of charged particle multiplicity in restricted intervals of phase space, specifically for $|y| \leq 2$ and $|y| \leq 1$. This complements our study of the charged particle multiplicity distributions of $g_{\text {incl. and }}$ uds jets in full phase space, presented in [6]. The distribution of charged multiplicity in restricted regions of phase space is more sensitive to the dynamics of multihadron production than the distribution in full phase space because it

\footnotetext{
7 The shape of the rapidity distribution is very sensitive to small changes in $\hat{r}$. If the thrust axis determined using the entire event is used to define rapidity, in addition to defining the $g_{\text {incl. }}$ hemisphere boundary, we find the agreement between the $y$ distributions of gg event hemispheres and $g_{\text {incl. }}$ jets to be somewhat less good, especially at small values $(y<0.2)$, than the agreement shown in Fig. 2a (see Sect. 6) for rapidity based on the sphericity axis, i.e. the sphericity axis determined using only those particles in the $g_{\text {incl. }}$ hemisphere is less influenced by soft particles in the opposite hemisphere than the thrust axis determined using the entire event. This is the motivation for our choice of $\hat{r}$
} 

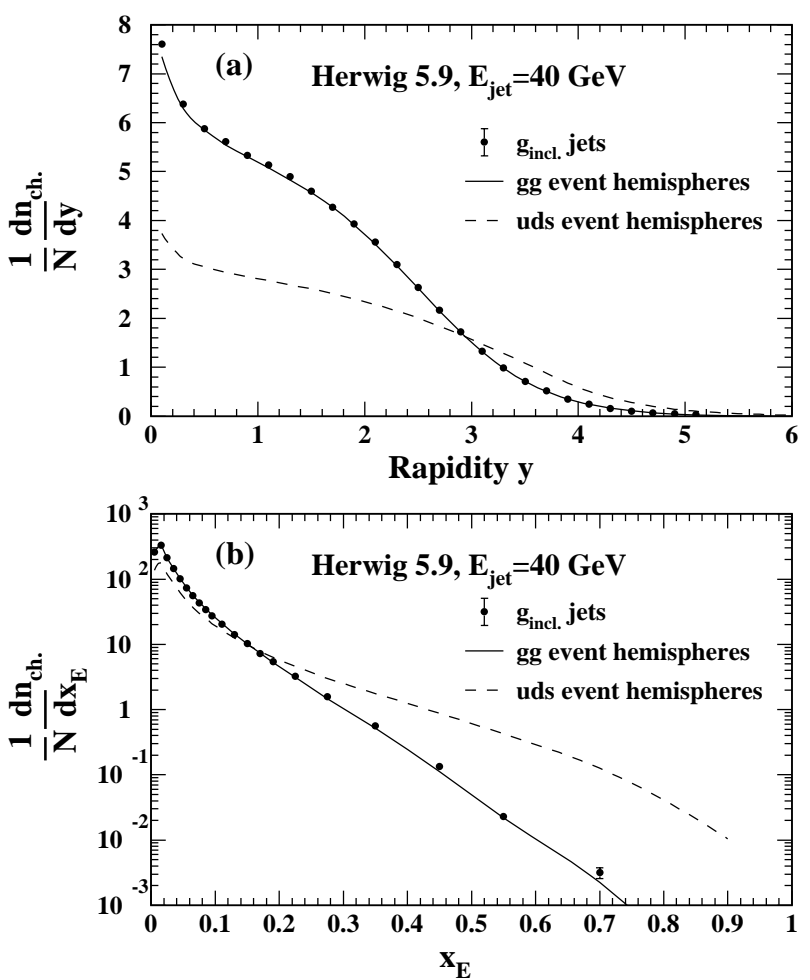

Fig. 2a,b. The prediction of the Herwig parton shower Monte Carlo event generator for the charged particle a rapidity and $\mathbf{b} x_{E}=E / E_{\text {jet }}$ distributions of $g_{\text {incl. }}$ gluon jets from $\mathrm{e}^{+} \mathrm{e}^{-}$annihilations, in comparison to the Herwig predictions for gg and uds event hemispheres. The jet energies are $40 \mathrm{GeV}$, corresponding to a c.m. energy of $91.2 \mathrm{GeV}$ for the generation of the $\mathrm{e}^{+} \mathrm{e}^{-} \rightarrow \mathrm{q} \overline{\mathrm{q}} \mathrm{g}_{\text {incl. }}$ events

is less affected by the constraints of overall charge and energy-momentum conservation.

With the exception of multiplicity, the distributions in this paper are normalized by the number of events in the sample. The multiplicity distributions are normalized to have unit area.

\section{Monte Carlo comparison of $g_{\text {incl. }}$ and $g g$ jets}

Our analysis of gluon jets is based on the premise that $\mathrm{g}_{\text {incl. }}$ jets from $\mathrm{e}^{+} \mathrm{e}^{-}$annihilations are equivalent to hemispheres of gg events produced from a color singlet point source, with the hemispheres defined by the plane perpendicular to the principal event axis. Although high energy gg events are not available experimentally, they may be generated using a QCD Monte Carlo event generator. The viability of our premise can be tested by comparing the Monte Carlo predictions for gg event hemispheres

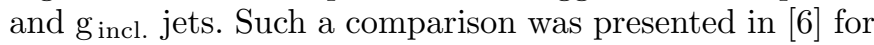
the charged particle multiplicity distribution in full phase space (see also [3]). In this section we extend this comparison to the distributions studied here.

The solid points in Fig. 2a show the prediction of the Herwig Monte Carlo multihadronic event generator [25], version 5.9, for the charged particle rapidity distribution

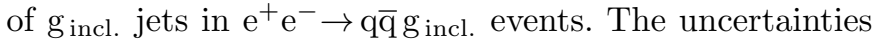
are statistical (these are too small to be visible). The parameter set we use is the same as that given in [23] for Herwig, version 5.8, except that the value of the cluster mass cutoff CLMAX has been increased from $3.40 \mathrm{GeV} / c^{2}$ to $3.75 \mathrm{GeV} / c^{2}$ to improve the model's description of the mean charged particle multiplicity $\left\langle n_{\mathrm{ch}}\right\rangle$ in inclusive hadronic $\mathrm{Z}^{0}$ decays [26]-[28]. The $\mathrm{e}^{+} \mathrm{e}^{-} \rightarrow \mathrm{q} \overline{\mathrm{q}} \mathrm{g}_{\text {incl. }}$ events were generated using a center-of-mass (c.m.) energy, $E_{\text {c.m. }}$, of $91.2 \mathrm{GeV}$ to correspond to the data. The $\mathrm{g}_{\text {incl. }}$ identification was performed using the same procedure as is described for the data in Sect. 3, except that the two quark

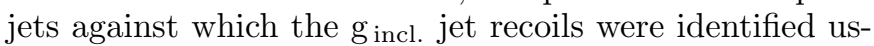
ing parton level Monte Carlo information as described in Sect. 3 rather than using displaced secondary vertices. In particular, the angular restrictions on the directions of the quark jets with respect to the thrust axis and to each other have been applied. The resulting mean energy of

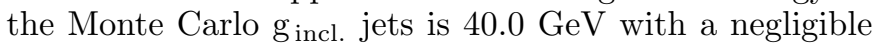
statistical uncertainty.

The solid curve in Fig. 2a shows the rapidity distribution predicted by Herwig for gg event hemispheres. The gg events were generated using a c.m. energy of $80.0 \mathrm{GeV}$ so that the hemisphere energies are the same as for the $\mathrm{g}_{\text {incl. }}$ jets. It is seen that the results for $\mathrm{g}_{\text {incl. }}$ jets and $\mathrm{gg}$ event hemispheres are almost indistinguishable. This establishes the validity of our technique to identify gluon jets in a manner which corresponds to point source production from a color singlet [3]. Similar agreement between the predicted rapidity distributions of $\mathrm{g}_{\text {incl. }}$ jets and $\mathrm{gg}$ event hemispheres is obtained if Jetset is used to generate the samples rather than Herwig, or if the JADE-E0 [29] or cone [30] jet finder is used to identify the quark jets for the $\mathrm{g}_{\text {incl. }}$ jet selection rather than the $k_{\perp}$ jet finder. This emphasizes the independence of our results from the choice of a jet finding algorithm. For purposes of comparison, the dashed curve in Fig. 2a shows the prediction of Herwig for uds event hemispheres, generated using the same c.m. energy as is used to generate the gg event sample.

The corresponding Monte Carlo comparison of the properties of $\mathrm{g}_{\text {incl. }}$ jets with those of $\mathrm{gg}$ and uds event hemispheres is shown in Fig. $2 \mathrm{~b}$ for the $x_{E}$ distribution, in Fig. 3 for the $\ln (p)$ and $p_{\mathrm{T}}(p<4 \mathrm{GeV} / c)$ distributions, and in Fig. 4 for the charged particle multiplicity distributions with $|y| \leq 2$ and $|y| \leq 1$. The results for the inclusive $p_{\mathrm{T}}$ distribution are qualitatively similar to those shown in Fig. $3 \mathrm{~b}$ for the $p_{\mathrm{T}}(p<4 \mathrm{GeV} / c)$ distribution and so we do not show them in addition. The results for $\mathrm{g}_{\text {incl. }}$ jets are seen to reproduce those of gg event hemispheres with good accuracy. In Fig. 4, a small shift is observable between the multiplicity distributions of $g_{\text {incl. }}$ and gg event hemispheres at intermediate values of multiplicity, with

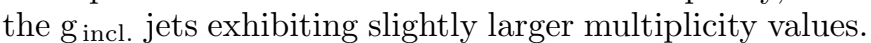
This shift is more pronounced for smaller rapidity intervals, i.e., it is more pronounced in Fig. 4b than in Fig. 4a (no such shift is visible for the charged particle multiplicity distribution in full phase space, see Fig. 1 in [6]). This

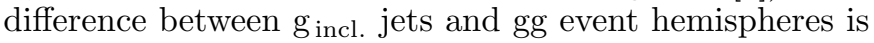



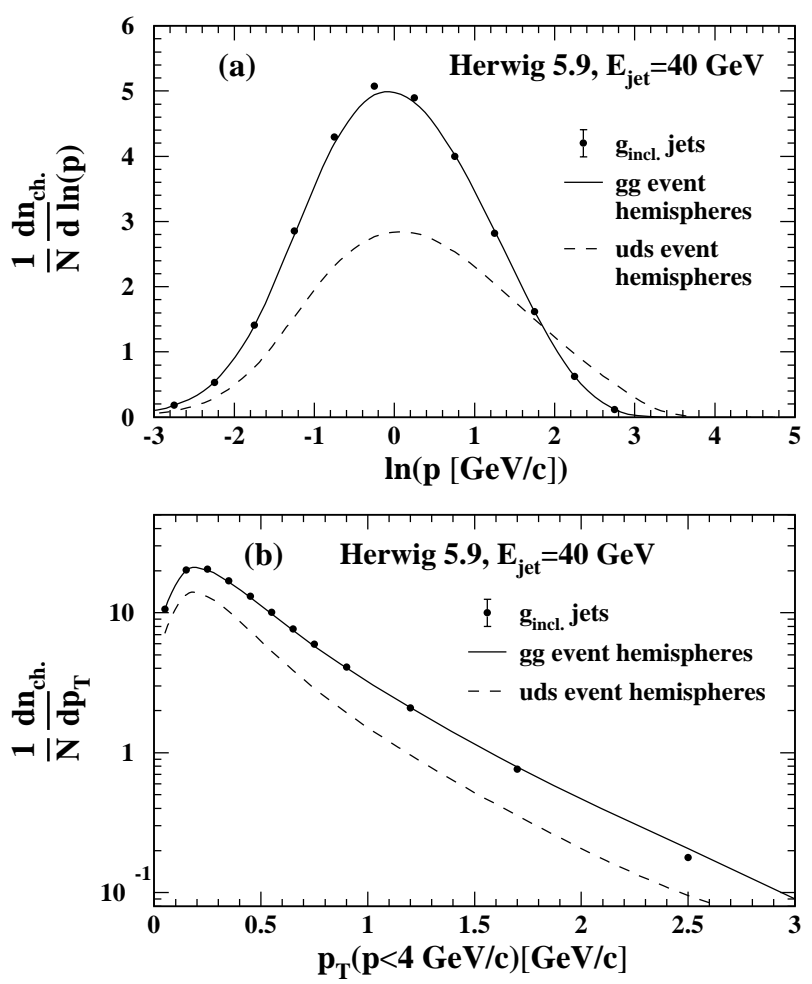

Fig. 3a,b. The prediction of the Herwig parton shower Monte Carlo event generator for the charged particle a $\ln (p)$ and b $p_{\mathrm{T}}(p<4 \mathrm{GeV} / c)$ distributions of $\mathrm{g}_{\text {incl. }}$ gluon jets from $\mathrm{e}^{+} \mathrm{e}^{-}$ annihilations, in comparison to the Herwig predictions for gg and uds event hemispheres. The jet energies are $40 \mathrm{GeV}$, corresponding to a c.m. energy of $91.2 \mathrm{GeV}$ for the generation of the $\mathrm{e}^{+} \mathrm{e}^{-} \rightarrow \mathrm{q} \overline{\mathrm{q}} \mathrm{g}_{\text {incl. }}$ events

negligible compared to the experimental statistical uncertainties (Sect. 9) or to the difference between the uds and

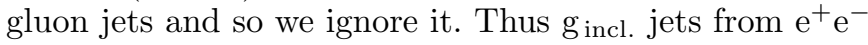
events have almost identical properties to gluon jets in gg events produced from a color singlet point source, as stipulated in the introduction.

\section{Corrections}

To correct the data for detector response and initial-state photon radiation, we generate events with the Jetset Monte Carlo and compare their properties with and without simulation of the detector and with and without initialstate radiation. The data are corrected to the hadron level. The hadron level does not include detector simulation or initial-state radiation and treats all charged and neutral particles with lifetimes greater than $3 \times 10^{-10} \mathrm{~s}$ as stable: hence charged particles from the decays of $\mathrm{K}_{\mathrm{S}}^{0}$ and weakly decaying hyperons are included in the corrected distributions. The corrections account not only for detector response and initial-state radiation but also for the background to the $\mathrm{g}_{\text {incl. }}$ and uds events. There is good agreement between the data and Monte Carlo for Monte Carlo samples which include background, initial-state radiation, detector simulation, and the same analysis proce-
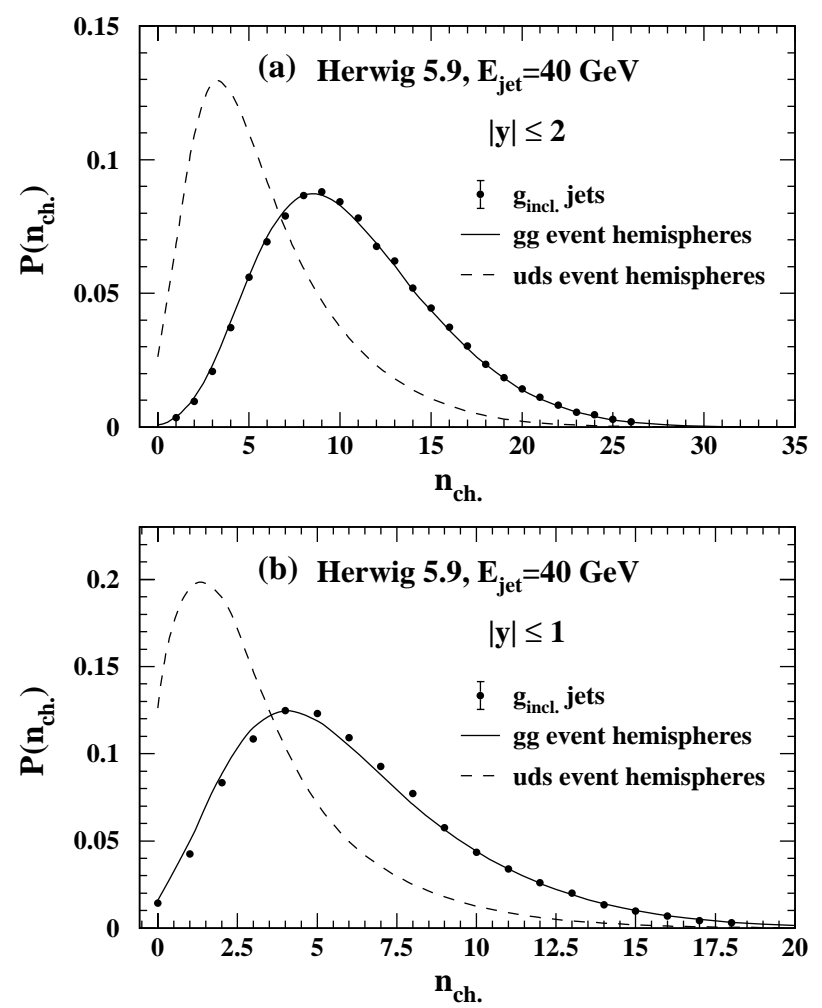

Fig. 4a,b. The prediction of the Herwig parton shower Monte Carlo event generator for the charged particle multiplicity distributions of $\mathrm{g}_{\text {incl. }}$ gluon jets from $\mathrm{e}^{+} \mathrm{e}^{-}$annihilations, in the rapidity intervals $\mathbf{a}|y| \leq 2$ and $\mathbf{b}|y| \leq 1$, in comparison to the Herwig predictions for gg and uds event hemispheres. The jet energies are $40 \mathrm{GeV}$, corresponding to a c.m. energy of $91.2 \mathrm{GeV}$ for the generation of the $\mathrm{e}^{+} \mathrm{e}^{-} \rightarrow \mathrm{q} \overline{\mathrm{q}} \mathrm{g}_{\text {incl. }}$ events

dures as are applied to the data. Furthermore, systematic shifts observed between Jetset and the data before the corrections are applied are also observed after corrections. For example, the mean charged particle multiplicity of $g_{\text {incl. }}$ jets in full phase space is $1 \%$ larger in Jetset than in the data before corrections and 3\% larger after corrections. Thus the corrections do not introduce a significant bias towards the predictions of Jetset.

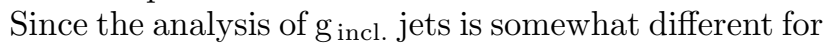
the 1991-92 and 1993-95 data samples (Sect. 3), separate corrections are determined for them. The corrected results from the two samples are consistent with each other to within their statistical uncertainties. The final gluon jet results are obtained by forming the weighted mean of the corrected 1991-92 and 1993-95 results.

The distributions of $y, x_{E}, \ln (p), p_{\mathrm{T}}$ and $p_{\mathrm{T}}(p<$ $4 \mathrm{GeV} / c)$ are corrected using bin-by-bin multiplicative factors which are determined as described in [5]. The corrections are typically in the range between 0.90 and 1.15 for both the $\mathrm{g}_{\text {incl. }}$ and uds jets. For the distributions of charged particle multiplicity with $|y| \leq 2$ and $|y| \leq 1$, we do not utilize simple bin-by-bin corrections since the Monte Carlo predicts considerable migration between multiplicity bins as a consequence of the detector response. Instead, the data are corrected in a two stage process using 
Table 1. The charged particle rapidity distribution, $|y|$, of $40.1 \mathrm{GeV} g_{\text {incl. }}$ gluon jets and $45.6 \mathrm{GeV}$ uds quark jets, and the ratio of $40.1 \mathrm{GeV} \mathrm{g}_{\text {incl. }}$ to $40.1 \mathrm{GeV}$ uds quark jets. The first uncertainty is statistical and the second is systematic. These data are displayed in Fig. 5

\begin{tabular}{cccc}
\hline$|y|$ & g incl. gluon jet & uds quark jet & Ratio \\
\hline $0.0-0.2$ & $7.42 \pm 0.35 \pm 0.70$ & $3.937 \pm 0.021 \pm 0.074$ & $1.97 \pm 0.09 \pm 0.20$ \\
$0.2-0.4$ & $6.37 \pm 0.31 \pm 0.59$ & $3.361 \pm 0.016 \pm 0.077$ & $1.97 \pm 0.10 \pm 0.18$ \\
$0.4-0.6$ & $5.86 \pm 0.26 \pm 0.48$ & $3.206 \pm 0.014 \pm 0.068$ & $1.90 \pm 0.09 \pm 0.17$ \\
$0.6-0.8$ & $5.39 \pm 0.29 \pm 0.61$ & $3.117 \pm 0.015 \pm 0.064$ & $1.80 \pm 0.10 \pm 0.21$ \\
$0.8-1.0$ & $5.31 \pm 0.26 \pm 0.61$ & $3.040 \pm 0.012 \pm 0.051$ & $1.82 \pm 0.09 \pm 0.21$ \\
$1.0-1.2$ & $5.40 \pm 0.28 \pm 0.65$ & $2.928 \pm 0.013 \pm 0.043$ & $1.92 \pm 0.10 \pm 0.23$ \\
$1.2-1.4$ & $5.30 \pm 0.25 \pm 0.54$ & $2.858 \pm 0.012 \pm 0.034$ & $1.93 \pm 0.09 \pm 0.20$ \\
$1.4-1.6$ & $4.86 \pm 0.21 \pm 0.52$ & $2.739 \pm 0.014 \pm 0.034$ & $1.84 \pm 0.08 \pm 0.20$ \\
$1.6-1.8$ & $4.39 \pm 0.20 \pm 0.42$ & $2.649 \pm 0.012 \pm 0.030$ & $1.72 \pm 0.08 \pm 0.17$ \\
$1.8-2.0$ & $3.98 \pm 0.21 \pm 0.32$ & $2.571 \pm 0.012 \pm 0.026$ & $1.60 \pm 0.09 \pm 0.13$ \\
$2.0-2.2$ & $3.47 \pm 0.21 \pm 0.28$ & $2.464 \pm 0.013 \pm 0.023$ & $1.46 \pm 0.09 \pm 0.12$ \\
$2.2-2.4$ & $3.06 \pm 0.21 \pm 0.23$ & $2.306 \pm 0.010 \pm 0.027$ & $1.38 \pm 0.10 \pm 0.11$ \\
$2.4-2.6$ & $2.85 \pm 0.18 \pm 0.22$ & $2.159 \pm 0.012 \pm 0.031$ & $1.38 \pm 0.09 \pm 0.11$ \\
$2.6-2.8$ & $2.00 \pm 0.18 \pm 0.28$ & $2.019 \pm 0.010 \pm 0.022$ & $1.04 \pm 0.09 \pm 0.15$ \\
$2.8-3.0$ & $1.84 \pm 0.13 \pm 0.24$ & $1.856 \pm 0.010 \pm 0.016$ & $1.05 \pm 0.08 \pm 0.14$ \\
$3.0-3.2$ & $1.22 \pm 0.13 \pm 0.25$ & $1.656 \pm 0.009 \pm 0.012$ & $0.79 \pm 0.08 \pm 0.16$ \\
$3.2-3.4$ & $0.96 \pm 0.11 \pm 0.16$ & $1.457 \pm 0.008 \pm 0.013$ & $0.72 \pm 0.08 \pm 0.12$ \\
$3.4-3.6$ & $0.63 \pm 0.09 \pm 0.17$ & $1.243 \pm 0.008 \pm 0.020$ & $0.56 \pm 0.08 \pm 0.16$ \\
$3.6-3.8$ & $0.62 \pm 0.08 \pm 0.12$ & $1.015 \pm 0.007 \pm 0.021$ & $0.69 \pm 0.08 \pm 0.13$ \\
$3.8-4.0$ & $0.32 \pm 0.06 \pm 0.11$ & $0.834 \pm 0.007 \pm 0.024$ & $0.43 \pm 0.09 \pm 0.15$ \\
$4.0-4.2$ & $0.185 \pm 0.043 \pm 0.076$ & $0.658 \pm 0.005 \pm 0.017$ & $0.32 \pm 0.08 \pm 0.13$ \\
$4.2-4.4$ & $0.169 \pm 0.047 \pm 0.070$ & $0.486 \pm 0.005 \pm 0.018$ & $0.41 \pm 0.11 \pm 0.16$ \\
$4.4-4.6$ & $0.100 \pm 0.034 \pm 0.046$ & $0.361 \pm 0.004 \pm 0.020$ & $0.32 \pm 0.11 \pm 0.15$ \\
$4.6-4.8$ & $0.046 \pm 0.026 \pm 0.046$ & $0.265 \pm 0.004 \pm 0.020$ & $0.21 \pm 0.11 \pm 0.20$ \\
$4.8-5.0$ & $0.048 \pm 0.020 \pm 0.031$ & $0.197 \pm 0.003 \pm 0.019$ & $0.29 \pm 0.13 \pm 0.19$ \\
$5.0-5.2$ & $0.013 \pm 0.018 \pm 0.023$ & $0.133 \pm 0.002 \pm 0.015$ & $0.12 \pm 0.17 \pm 0.18$ \\
$5.2-5.4$ & - & $0.09549 \pm 0.0026 \pm 0.0098$ & - \\
$5.4-5.6$ & $0.0051 \pm 0.0082 \pm 0.0055$ & $0.0626 \pm 0.0019 \pm 0.0054$ & $0.10 \pm 0.15 \pm 0.09$ \\
$5.6-5.8$ & - & $0.0453 \pm 0.0014 \pm 0.0034$ & - \\
$5.8-6.0$ & - & $0.0290 \pm 0.0012 \pm 0.0020$ & - \\
\hline & & &
\end{tabular}

the method described in [6]. In the first stage, the data are corrected for experimental acceptance, resolution, and secondary electromagnetic and hadronic interactions using an unfolding matrix [31]. In the second stage, the data are corrected for background, geometric event acceptance, and the effects of initial-state radiation using bin-by-bin factors. More details are given in [6]. As an indication of the overall size of the corrections, Jetset predicts the

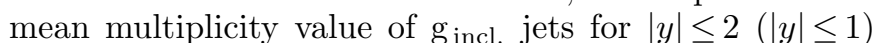
to be $11 \%(13 \%)$ larger at the hadron level than it is at the level which includes background, initial-state radiation, detector simulation, and the experimental selection criteria. The corresponding difference for uds jets is $0 \%$ $(+1 \%)$.

\section{Systematic uncertainties}

To evaluate systematic uncertainties, the analysis was repeated with the following changes relative to the standard analysis. There were no significant changes in the number of selected events or in the estimated purities of the gluon and quark jet samples compared to the standard results ( $439 \mathrm{~g}_{\text {incl. }}$ jets with $81.9 \%$ purity and 107104 uds jets with $86.4 \%$ purity) unless otherwise noted.

1. Charged tracks alone were used for the data and for the Monte Carlo samples with detector simulation, rather than charged tracks plus electromagnetic clusters; the number of selected $\mathrm{g}_{\text {incl. }}$ jets decreased to 327 . As an additional check on the track selection, the minimum momentum of charged tracks was increased from from $0.10 \mathrm{GeV} / c$ to $0.25 \mathrm{GeV} / c$. 
2. Herwig was used to determine the corrections for background, detector response and initial-state radiation, rather than Jetset.

3. The gluon jet selection was performed using the JADEE0 and cone jet finders to define the tagged quark jets, rather than the $k_{\perp}$ jet finder; for the analysis based on the cone jet finder, the number of $\mathrm{g}_{\text {incl. jets dropped }}$ to 346 while their estimated purity decreased to $73.2 \%$.

4. The geometric conditions for the gluon jet selection were varied, first by requiring the angle between the two jets in the tagged hemisphere to exceed $50^{\circ}$, rather than $70^{\circ}$, and second by requiring the two tagged quark jets to lie within $65^{\circ}$ of the thrust axis, rather than $70^{\circ}$. For the first of these conditions, the $g_{\text {incl. sample in- }}$ creased to 583 jets while its estimated purity decreased to $73.5 \%$; for the second of these conditions, the $g_{\text {incl. }}$ sample decreased to 383 jets.

5. Secondary vertices used to tag quark jets for the $\mathrm{g}_{\text {incl. }}$ identification were required to have decay lengths which satisfied $L / \sigma_{L}>5.0$, rather than $L / \sigma_{L}>3.5$, for the 1991-92 data, and $L / \sigma_{L}>7.0$, rather than $L / \sigma_{L}>$ 5.0 , for the 1993-95 data; the $g_{\text {incl. sample decreased to }}$ 268 jets while its estimated purity increased to $84.9 \%$.

6. Tracks selected for the uds tagging procedure were required to have a signed impact parameter which satisfied $b / \sigma_{b}>1.5$, rather than $b / \sigma_{b}>2.5$; the uds sample decreased to 49396 jets while its estimated purity increased to $89.9 \%$. As an additional check on the track selection for the uds tagging procedure, tracks used for this procedure were required to satisfy the following criteria: (i) the maximum distance of closest approach of the track to the primary event vertex in the $r$ - $\phi$ plane was $5.0 \mathrm{~cm}$, rather than $0.3 \mathrm{~cm}$, (ii) no requirement was placed on the uncertainty of the distance of closest approach of the track to the event vertex, rather than requiring this uncertainty to be less than $0.1 \mathrm{~cm}$ in the $r$ - $\phi$ plane, and (iii) the minimum momentum was $0.1 \mathrm{GeV} / c$ rather than $0.5 \mathrm{GeV} / c$; the uds sample decreased to 70826 jets.

7. For the ratios of the gluon to quark jets results (see Sect. 9), the energy to which the quark jet results were corrected was varied by the total uncertainty of the

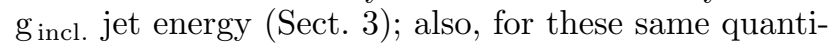
ties, the correction factors to account for the difference

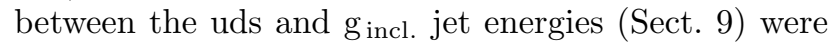
varied by their uncertainties.

The differences between the standard results and those found using each of these conditions were used to define symmetric systematic uncertainties. For items 1, 3, 4, 6 and 7 , the larger of the two described differences with respect to the standard result was assigned as the systematic uncertainty. For item 2, the difference with respect to the standard result was multiplied by $2 / \sqrt{12}$ for the following reason. Jetset describes the basic experimental distributions in this paper very well (as already stated in Sect. 7) and in this sense its results represent a central value compared to the results of other Monte Carlo simulations. In contrast, Herwig disagrees with the data for some basic
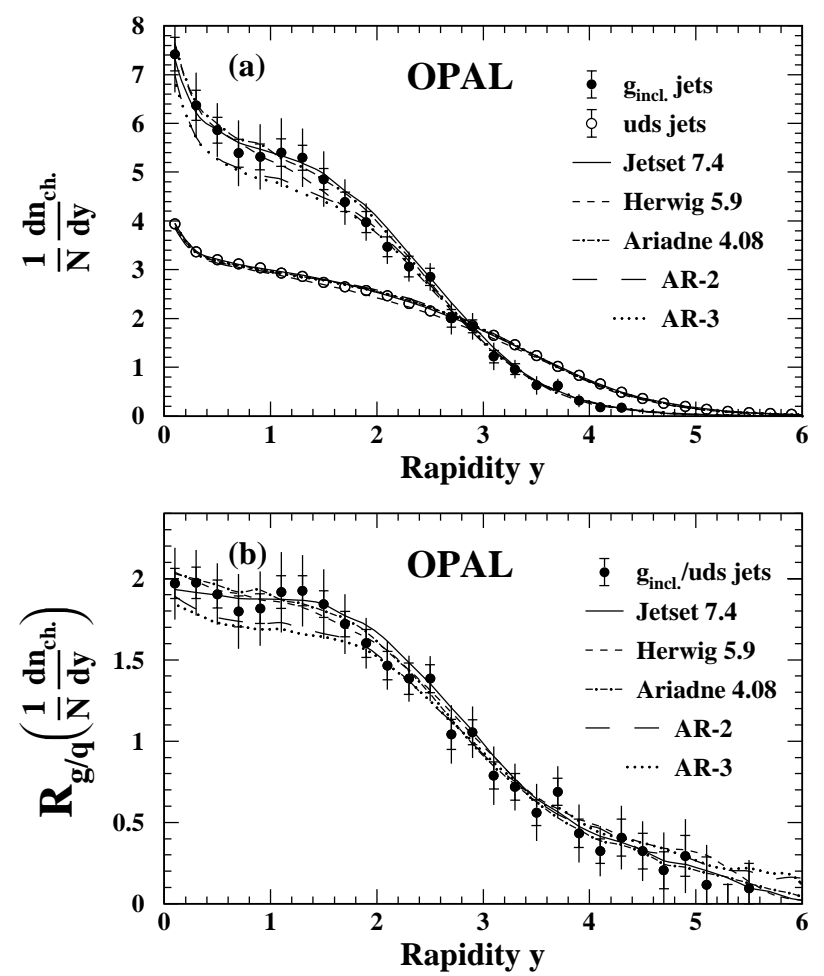

Fig. 5. a Corrected distributions of charged particle rapidity, $y$, for $40.1 \mathrm{GeV} \mathrm{g}_{\text {incl. }}$ gluon jets and $45.6 \mathrm{GeV}$ uds quark jets. b The ratio of the gluon to quark jet rapidity distributions for 40.1 GeV jets. The total uncertainties are shown by vertical lines. The experimental statistical uncertainties are indicated by small horizontal bars. (The uncertainties are too small to be seen for the uds jets.) The predictions of various parton shower Monte Carlo event generators are also shown. These data are tabulated in Table 1

distributions, especially for uds jets. ${ }^{8}$ Thus, the results of Herwig represent an extreme choice for this analysis. The factor of $2 / \sqrt{12}$ converts the difference between an extreme and central value into a dispersion, i.e. into a more realistic estimate of the uncertainty related to the model dependence of the corrections for detector response.

The systematic uncertainty evaluated for each bin of the differential distributions (Figs. 5-10, see Sect. 9) was averaged with the results from its two neighbors to reduce the effect of bin-to-bin fluctuations (the single neighbor was used for bins on the endpoints of the distributions). The uncertainties were added in quadrature to define the total systematic uncertainty. The largest systematic terms for the $\mathrm{g}_{\text {incl. }}$ jet measurements generally arose about equally from items $1,3,4$ and 5 in the above list.

\footnotetext{
${ }^{8}$ For example, the mean charged particle multiplicity of uds jet hemispheres is measured to be $10.10 \pm 0.18$ (stat.+syst.) [6], compared to predictions of 9.67 and 10.03 for our tuned versions of Herwig and Jetset, respectively: this represents a difference of 2.4 standard deviations for Herwig but of only 0.4 standard deviations for Jetset. The low value of the Herwig prediction for multiplicity in uds jets is reflected in some of the basic distributions of our study, such as the uds jet rapidity distribution shown in Fig. 5a (Sect. 9)
} 

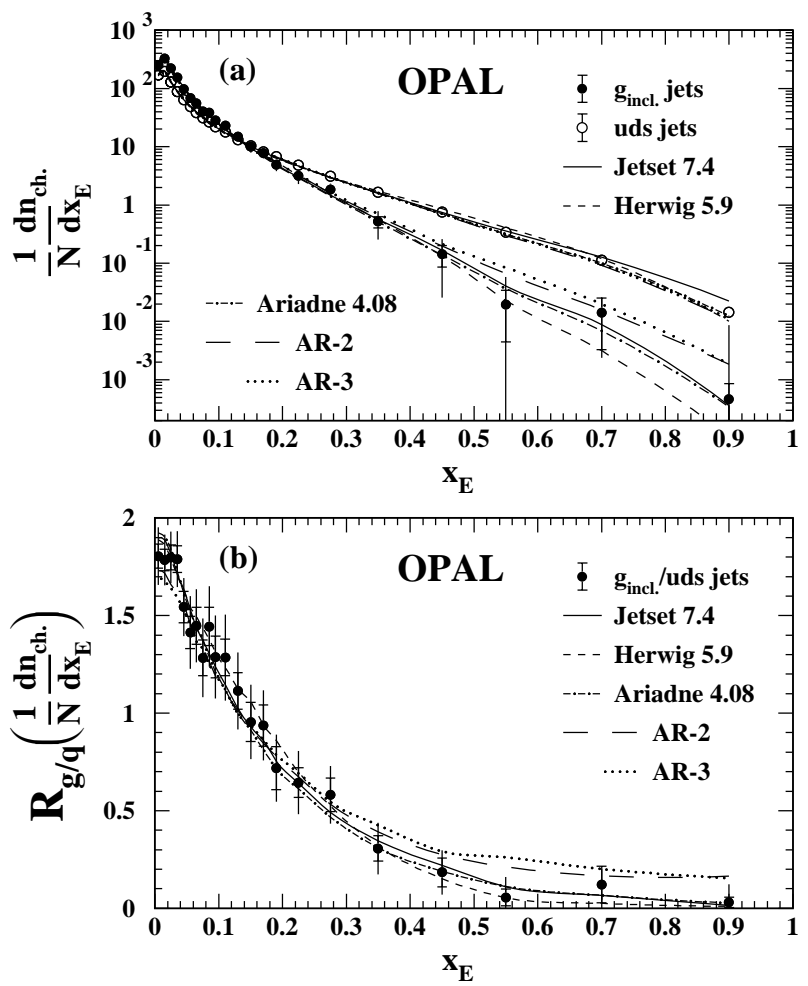

Fig. 6. a Corrected distributions of charged particle scaled en-

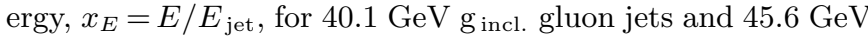
uds quark jets. $\mathbf{b}$ The ratio of the gluon to quark jet $x_{E}$ distributions for $40.1 \mathrm{GeV}$ jets. The total uncertainties are shown by vertical lines. The experimental statistical uncertainties are indicated by small horizontal bars. (The uncertainties are too small to be seen for the uds jets.) The predictions of various parton shower Monte Carlo event generators are also shown. These data are tabulated in Table 2

The largest systematic terms for the uds jet measurements generally arose about equally from items 2 and 6 . For the ratios of the gluon to quark jet measurements, the largest systematic terms generally arose from items 1, 3 and 5 .

As an additional systematic check, events selected for the $\mathrm{g}_{\text {incl. }}$ analysis were required to have c.m. energies within $100 \mathrm{MeV}$ of the $\mathrm{Z}^{0}$ peak. This restriction eliminated $12 \%$ of the events from the $\mathrm{g}_{\text {incl. }}$ sample and resulted in insignificant changes to the measured $\mathrm{g}_{\text {incl. }}$ jet properties.

\section{Results}

The corrected distributions of $y, x_{E}, \ln (p), p_{\mathrm{T}}$ and $p_{\mathrm{T}}(p<$ $4 \mathrm{GeV} / c$ ) are shown in the top portions of Figs. 5-9. The corresponding results for charged particle multiplicity with $|y| \leq 2$ and $|y| \leq 1$ are shown in Fig. 10. Numerical values for these data are provided in Tables 1-7. The vertical lines on the data points show the total uncertainties, with statistical and systematic terms added in quadrature. The small horizontal lines indicate the size of the experimental statistical uncertainties. Our results for the charged particle uds fragmentation function (Fig. 6 and Table 2) are consistent with those presented in [32]. The
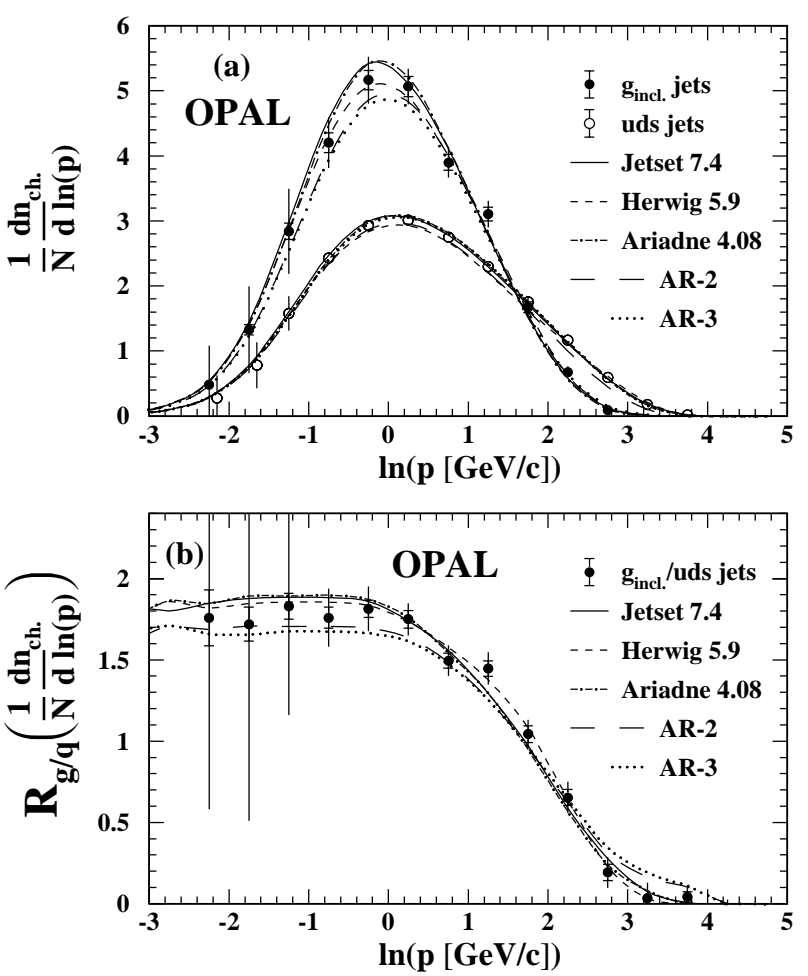

Fig. 7. a Corrected distributions of the logarithm of charged particle momentum, $\ln (p)$, for $40.1 \mathrm{GeV} \mathrm{g}_{\text {incl. }}$ gluon jets and $45.6 \mathrm{GeV}$ uds quark jets. $\mathbf{b}$ The ratio of the gluon to quark jet $\ln (p)$ distributions for $40.1 \mathrm{GeV}$ jets. The total uncertainties are shown by vertical lines. The experimental statistical uncertainties are indicated by small horizontal bars. (The statistical uncertainties are too small to be seen for the uds jets.) The predictions of various parton shower Monte Carlo event generators are also shown. These data are tabulated in Table 3

matrix correction technique employed for the multiplicity distributions in Fig. 10 introduces correlations between bins. These correlations are generally strong between a bin and its nearest one or two neighbors on either side but can extend with smaller strength to four or five bins away. The correlations smooth out bin-to-bin statistical fluctuations. This effect is particularly noticeable for the gluon jet distributions in Fig. 10 because of the relatively small number of events in the $\mathrm{g}_{\text {incl. }}$ jet sample.

For the $y, x_{E}, \ln (p), p_{\mathrm{T}}$ and $p_{\mathrm{T}}(p<4 \mathrm{GeV} / c)$ distributions, we also determine ratios between the gluon and quark jet measurements since common systematic uncertainties will partially cancel. Before forming these ratios, we account for the different energies of the two samples: the gluon jets have a mean energy of $40.1 \mathrm{GeV}$ whereas the uds jets have a mean energy of $45.6 \mathrm{GeV}$. To correct the quark jets for this difference in energy, we employ multiplicative factors determined bin-by-bin using Jetset. As a systematic check, we also determine the corrections predicted by Herwig. Since the energy difference between the $\mathrm{g}_{\text {incl. }}$ and uds jets is only $5.5 \mathrm{GeV}$, these corrections are small: they typically lie between 0.95 and 1.01 . The bottom portions of Figs. 5-9 show the ratios of the 
Table 2. The charged particle scaled energy, $x_{E}=E / E_{\text {jet }}$, of $40.1 \mathrm{GeV}$ g incl. gluon jets and $45.6 \mathrm{GeV}$ uds quark jets, and the ratio of $40.1 \mathrm{GeV} \mathrm{g}_{\text {incl. }}$ to $40.1 \mathrm{GeV}$ uds quark jets. The first uncertainty is statistical and the second is systematic. These data are displayed in Fig. 6

\begin{tabular}{cccc}
\hline$x_{E}$ & g incl. gluon jet & uds quark jet & Ratio \\
\hline $0.00-0.01$ & $248 \pm 9 \pm 21$ & $170.1 \pm 0.5 \pm 1.7$ & $1.80 \pm 0.06 \pm 0.13$ \\
$0.01-0.02$ & $328 \pm 10 \pm 18$ & $196.7 \pm 0.6 \pm 1.5$ & $1.79 \pm 0.05 \pm 0.12$ \\
$0.02-0.03$ & $222 \pm 8 \pm 16$ & $128.7 \pm 0.4 \pm 1.4$ & $1.80 \pm 0.06 \pm 0.12$ \\
$0.03-0.04$ & $155 \pm 6 \pm 11$ & $87.7 \pm 0.3 \pm 1.1$ & $1.79 \pm 0.07 \pm 0.13$ \\
$0.04-0.05$ & $97.7 \pm 5.0 \pm 8.6$ & $63.84 \pm 0.25 \pm 0.92$ & $1.54 \pm 0.08 \pm 0.13$ \\
$0.05-0.06$ & $67.9 \pm 4.0 \pm 7.9$ & $48.31 \pm 0.24 \pm 0.58$ & $1.41 \pm 0.08 \pm 0.17$ \\
$0.06-0.07$ & $55.4 \pm 3.6 \pm 6.2$ & $38.44 \pm 0.16 \pm 0.50$ & $1.45 \pm 0.09 \pm 0.16$ \\
$0.07-0.08$ & $40.1 \pm 2.8 \pm 6.0$ & $31.22 \pm 0.18 \pm 0.42$ & $1.28 \pm 0.09 \pm 0.18$ \\
$0.08-0.09$ & $38.2 \pm 2.6 \pm 4.7$ & $26.37 \pm 0.17 \pm 0.47$ & $1.44 \pm 0.10 \pm 0.18$ \\
$0.09-0.10$ & $28.5 \pm 2.4 \pm 4.0$ & $22.04 \pm 0.13 \pm 0.28$ & $1.29 \pm 0.11 \pm 0.18$ \\
$0.10-0.12$ & $23.0 \pm 1.7 \pm 3.6$ & $17.79 \pm 0.09 \pm 0.22$ & $1.29 \pm 0.09 \pm 0.20$ \\
$0.12-0.14$ & $14.9 \pm 1.2 \pm 2.3$ & $13.238 \pm 0.075 \pm 0.096$ & $1.11 \pm 0.09 \pm 0.17$ \\
$0.14-0.16$ & $10.0 \pm 1.0 \pm 1.8$ & $10.44 \pm 0.08 \pm 0.11$ & $0.95 \pm 0.10 \pm 0.16$ \\
$0.16-0.18$ & $7.7 \pm 0.9 \pm 1.3$ & $8.231 \pm 0.061 \pm 0.092$ & $0.94 \pm 0.11 \pm 0.15$ \\
$0.18-0.20$ & $4.94 \pm 0.76 \pm 0.86$ & $6.776 \pm 0.056 \pm 0.086$ & $0.72 \pm 0.11 \pm 0.13$ \\
$0.20-0.25$ & $3.15 \pm 0.37 \pm 0.77$ & $4.829 \pm 0.035 \pm 0.053$ & $0.64 \pm 0.08 \pm 0.14$ \\
$0.25-0.30$ & $1.85 \pm 0.27 \pm 0.38$ & $3.105 \pm 0.022 \pm 0.049$ & $0.58 \pm 0.09 \pm 0.12$ \\
$0.30-0.40$ & $0.52 \pm 0.11 \pm 0.24$ & $1.655 \pm 0.012 \pm 0.039$ & $0.31 \pm 0.07 \pm 0.11$ \\
$0.40-0.50$ & $0.14 \pm 0.06 \pm 0.10$ & $0.757 \pm 0.007 \pm 0.036$ & $0.184 \pm 0.075 \pm 0.084$ \\
$0.50-0.60$ & $0.019 \pm 0.015 \pm 0.032$ & $0.339 \pm 0.006 \pm 0.017$ & $0.056 \pm 0.043 \pm 0.087$ \\
$0.60-0.80$ & $0.014 \pm 0.011 \pm 0.012$ & $0.1118 \pm 0.0026 \pm 0.0070$ & $0.121 \pm 0.093 \pm 0.078$ \\
$0.80-1.00$ & $0.0005 \pm 0.0004 \pm 0.008$ & $0.0143 \pm 0.0009 \pm 0.0026$ & $0.030 \pm 0.026 \pm 0.092$ \\
\hline & & &
\end{tabular}

Table 3. The logarithm of charged particle momentum, $\ln (p)$, of $40.1 \mathrm{GeV} g_{\text {incl. gluon jets }}$ and $45.6 \mathrm{GeV}$ uds quark jets, and the ratio of $40.1 \mathrm{GeV} \mathrm{g}_{\text {incl. }}$ to $40.1 \mathrm{GeV}$ uds quark jets. The first uncertainty is statistical and the second is systematic. These data are displayed in Fig. 7

\begin{tabular}{cccc}
\hline $\ln (p)$ & $\mathrm{g}_{\text {incl. }}$ gluon jet & uds quark jet & Ratio \\
\hline$-2.5--2.0$ & $0.48 \pm 0.05 \pm 0.60$ & $0.27 \pm 0.01 \pm 0.35$ & $1.8 \pm 0.2 \pm 1.2$ \\
$-2.0--1.5$ & $1.33 \pm 0.08 \pm 0.67$ & $0.78 \pm 0.01 \pm 0.35$ & $1.7 \pm 0.1 \pm 1.2$ \\
$-1.5--1.0$ & $2.84 \pm 0.12 \pm 0.64$ & $1.58 \pm 0.01 \pm 0.26$ & $1.83 \pm 0.08 \pm 0.67$ \\
$-1.0--0.5$ & $4.20 \pm 0.15 \pm 0.36$ & $2.431 \pm 0.008 \pm 0.021$ & $1.76 \pm 0.06 \pm 0.17$ \\
$-0.5-0.0$ & $5.17 \pm 0.15 \pm 0.33$ & $2.925 \pm 0.009 \pm 0.026$ & $1.81 \pm 0.05 \pm 0.13$ \\
$0.0-0.5$ & $5.07 \pm 0.15 \pm 0.22$ & $3.012 \pm 0.008 \pm 0.028$ & $1.749 \pm 0.053 \pm 0.084$ \\
$0.5-1.0$ & $3.90 \pm 0.12 \pm 0.20$ & $2.749 \pm 0.009 \pm 0.029$ & $1.495 \pm 0.047 \pm 0.080$ \\
$1.0-1.5$ & $3.11 \pm 0.10 \pm 0.18$ & $2.299 \pm 0.007 \pm 0.023$ & $1.447 \pm 0.048 \pm 0.087$ \\
$1.5-2.0$ & $1.67 \pm 0.08 \pm 0.13$ & $1.757 \pm 0.006 \pm 0.016$ & $1.043 \pm 0.052 \pm 0.073$ \\
$2.0-2.5$ & $0.670 \pm 0.050 \pm 0.078$ & $1.167 \pm 0.005 \pm 0.012$ & $0.653 \pm 0.049 \pm 0.083$ \\
$2.5-3.0$ & $0.091 \pm 0.024 \pm 0.036$ & $0.592 \pm 0.003 \pm 0.012$ & $0.192 \pm 0.051 \pm 0.080$ \\
$3.0-3.5$ & $0.004 \pm 0.003 \pm 0.023$ & $0.1788 \pm 0.0017 \pm 0.0083$ & $0.032 \pm 0.023 \pm 0.093$ \\
$3.5-4.0$ & $0.0002 \pm 0.0002 \pm 0.0046$ & $0.0138 \pm 0.0006 \pm 0.0028$ & $0.041 \pm 0.032 \pm 0.059$ \\
\hline
\end{tabular}


Table 4. The charged particle transverse momentum with respect to the jet axis, $p_{\mathrm{T}}$, of $40.1 \mathrm{GeV} \mathrm{g}_{\text {incl. }}$ gluon jets and $45.6 \mathrm{GeV}$ uds quark jets, and the ratio of $40.1 \mathrm{GeV} \mathrm{g}_{\text {incl. }}$ to $40.1 \mathrm{GeV}$ uds quark jets. The first uncertainty is statistical and the second is systematic. These data are displayed in Fig. 8

\begin{tabular}{cccc}
\hline$p_{\mathrm{T}}$ & $\mathrm{g}_{\text {incl. gluon jet }}$ & uds quark jet & Ratio \\
\hline $0.0-0.1$ & $9.73 \pm 0.47 \pm 0.92$ & $7.38 \pm 0.03 \pm 0.24$ & $1.359 \pm 0.065 \pm 0.093$ \\
$0.1-0.2$ & $21.3 \pm 0.6 \pm 1.0$ & $15.16 \pm 0.04 \pm 0.19$ & $1.445 \pm 0.044 \pm 0.085$ \\
$0.2-0.3$ & $20.9 \pm 0.8 \pm 1.2$ & $15.72 \pm 0.04 \pm 0.15$ & $1.369 \pm 0.051 \pm 0.081$ \\
$0.3-0.4$ & $17.3 \pm 0.6 \pm 1.1$ & $13.02 \pm 0.04 \pm 0.10$ & $1.377 \pm 0.050 \pm 0.093$ \\
$0.4-0.5$ & $14.04 \pm 0.58 \pm 0.97$ & $10.02 \pm 0.03 \pm 0.11$ & $1.46 \pm 0.06 \pm 0.10$ \\
$0.5-0.6$ & $11.37 \pm 0.50 \pm 0.85$ & $7.571 \pm 0.024 \pm 0.098$ & $1.57 \pm 0.07 \pm 0.13$ \\
$0.6-0.7$ & $8.82 \pm 0.45 \pm 0.92$ & $5.758 \pm 0.022 \pm 0.068$ & $1.62 \pm 0.08 \pm 0.18$ \\
$0.7-0.8$ & $7.67 \pm 0.40 \pm 0.90$ & $4.391 \pm 0.024 \pm 0.052$ & $1.87 \pm 0.10 \pm 0.21$ \\
$0.8-1.0$ & $5.42 \pm 0.23 \pm 0.56$ & $2.989 \pm 0.012 \pm 0.042$ & $1.97 \pm 0.08 \pm 0.18$ \\
$1.0-1.4$ & $2.64 \pm 0.13 \pm 0.37$ & $1.590 \pm 0.007 \pm 0.032$ & $1.84 \pm 0.09 \pm 0.30$ \\
$1.4-2.0$ & $1.24 \pm 0.07 \pm 0.19$ & $0.680 \pm 0.004 \pm 0.013$ & $2.08 \pm 0.12 \pm 0.34$ \\
$2.0-3.0$ & $0.36 \pm 0.03 \pm 0.15$ & $0.2497 \pm 0.0018 \pm 0.0054$ & $1.71 \pm 0.16 \pm 0.53$ \\
$3.0-4.0$ & $0.109 \pm 0.017 \pm 0.041$ & $0.0903 \pm 0.0010 \pm 0.0028$ & $1.51 \pm 0.23 \pm 0.46$ \\
$4.0-6.0$ & $0.029 \pm 0.005 \pm 0.022$ & $0.0289 \pm 0.0004 \pm 0.0015$ & $1.34 \pm 0.22 \pm 0.73$ \\
$6.0-8.0$ & $0.0036 \pm 0.0015 \pm 0.0065$ & $0.00860 \pm 0.00016 \pm 0.00083$ & $0.60 \pm 0.25 \pm 1.14$ \\
$8.0-10.0$ & $0.0035 \pm 0.0002 \pm 0.0041$ & $0.00304 \pm 0.00010 \pm 0.00051$ & $1.75 \pm 0.11 \pm 1.50$ \\
\hline
\end{tabular}

gluon to quark jet results, corresponding to jet energies of $40.1 \mathrm{GeV}$. Numerical values for these ratio measurements are included in Tables 1-5.

\subsection{Mean multiplicity ratio at small rapidities}

A striking feature of our results is the nearly factor of two difference between the mean multiplicities of gluon and quark jets at small rapidities and energies (see Figs. 5b and $6 \mathrm{~b})$. As a measure of this difference, we determine the ratio, $r_{\text {ch. }}$, of the mean gluon to quark jet charged particle multiplicity for $|y| \leq 1$. Our measurement of this ratio is

$$
r_{\text {ch. }}(|y| \leq 1)=1.919 \pm 0.047 \text { (stat.) } \pm 0.095 \text { (syst.) }
$$

For this ratio, the quark jet result has been corrected for the small difference in energy between the gluon and quark jets in the manner described above. The corresponding result for $|y| \leq 2$ is $r_{\text {ch. }}(|y| \leq 2)=1.852 \pm 0.034$ (stat.) \pm 0.077 (syst.). For purposes of comparison, we also report our measurement of $r_{\mathrm{ch}}$. in full phase space. This result, $r_{\text {ch. }}=1.514 \pm 0.019$ (stat.) \pm 0.034 (syst.), agrees well with our previous measurements $[5,6]$ and with recent QCD calculations of this quantity [33]. These results are summarized in Table 8.

For completeness, we also update our measurement of the mean charged particle multiplicity in $\mathrm{g}_{\text {incl. }}$ jets. We obtain $\left\langle n_{\text {ch. }}\right\rangle_{\mathrm{g}_{\text {incl. }}}=14.28 \pm 0.18$ (stat.) \pm 0.31 (syst.), in agreement with our earlier results $[5,6]$ but with a reduced uncertainty. The corresponding results for $|y| \leq 2$ and $|y| \leq 1$ are included in the bottom rows of Tables 6 and 7 .

The emphasis in the current study is on the multiplicity of soft particles in jets. In contrast, we previously studied the total charged multiplicity in gluon and quark jets $[5,6]$. For soft particles, i.e. particles with energies $E<<E_{\text {jet }}$, QCD predicts that the mean multiplicities in gluon and quark jets differ by a factor of $r=\mathrm{C}_{\mathrm{A}} / \mathrm{C}_{\mathrm{F}}=2.25$ $[34,35]$. Because our experimental definition of jets corresponds to the theoretical one, our result (relation (3)) provides the most direct test of this prediction to date. Nonetheless, the QCD prediction refers to partons whereas the measurement is based on hadrons. Furthermore, the QCD result does not account for energy-momentum conservation or higher order perturbative terms. These latter corrections are believed to be negligible in the asymptotic limit $E<<E_{\text {jet }}[35]$. Thus the directness of our test is limited only by hadronization effects and the extent to which the asymptotic condition is satisfied by our data.

To demonstrate the correspondence between our experimental variable (3) and $r$ as it is defined for analytic calculations, and to assess the origin of the remaining difference between our measurement $\left(r_{\mathrm{ch}} \approx 1.92\right.$ for $\left.|y| \leq 1\right)$ and the QCD prediction $(r=2.25)$, we examined the predictions of the Herwig Monte Carlo at the hadron and parton levels and for $E_{\text {jet }}=40.1 \mathrm{GeV}$ (as in our analysis) and $E_{\text {jet }}=5 \mathrm{TeV}$. The parton level results are obtained using the final-state partons, i.e., those which are present after termination of the parton shower. Herwig incorporates exact energy-momentum conservation, higher order perturbative terms up to and beyond the next-to-next-toleading order, a hadronization model, and exhibits the correct QCD asymptotic behavior as the c.m. energy becomes large (see, for example, Fig. 2 in [5]). ${ }^{9}$ Herwig is thus well suited to compare both to our data and to the asymp-

\footnotetext{
${ }^{9}$ In contrast, Jetset does not exhibit the correct asymptotic behavior [5]
} 
Table 5. The charged particle transverse momentum with respect to the jet axis for particles with momentum $p<4 \mathrm{GeV} / c, p_{\mathrm{T}}(p<4 \mathrm{GeV} / c)$, of $40.1 \mathrm{GeV} \mathrm{g}_{\text {incl. }}$ gluon jets and $45.6 \mathrm{GeV}$ uds quark jets, and the ratio of $40.1 \mathrm{GeV} \mathrm{g}_{\text {incl. }}$ to $40.1 \mathrm{GeV}$ uds quark jets. The first uncertainty is statistical and the second is systematic. These data are displayed in Fig. 9

\begin{tabular}{cccc}
\hline$p_{\mathrm{T}}(p<4 \mathrm{GeV} / c)$ & $\mathrm{g}_{\text {incl. gluon jet }}$ & uds quark jet & Ratio \\
\hline $0.0-0.1$ & $9.50 \pm 0.46 \pm 0.97$ & $6.91 \pm 0.03 \pm 0.22$ & $1.41 \pm 0.07 \pm 0.13$ \\
$0.1-0.2$ & $20.8 \pm 0.6 \pm 1.0$ & $13.85 \pm 0.04 \pm 0.16$ & $1.53 \pm 0.05 \pm 0.11$ \\
$0.2-0.3$ & $20.2 \pm 0.8 \pm 1.2$ & $13.86 \pm 0.04 \pm 0.13$ & $1.49 \pm 0.06 \pm 0.11$ \\
$0.3-0.4$ & $16.6 \pm 0.6 \pm 1.2$ & $10.94 \pm 0.04 \pm 0.10$ & $1.55 \pm 0.06 \pm 0.13$ \\
$0.4-0.5$ & $13.3 \pm 0.6 \pm 1.1$ & $8.049 \pm 0.03 \pm 0.11$ & $1.70 \pm 0.07 \pm 0.16$ \\
$0.5-0.6$ & $10.1 \pm 0.5 \pm 1.1$ & $5.836 \pm 0.023 \pm 0.091$ & $1.79 \pm 0.09 \pm 0.23$ \\
$0.6-0.7$ & $7.7 \pm 0.4 \pm 1.0$ & $4.301 \pm 0.020 \pm 0.068$ & $1.86 \pm 0.11 \pm 0.26$ \\
$0.7-0.8$ & $6.66 \pm 0.38 \pm 0.90$ & $3.169 \pm 0.021 \pm 0.052$ & $2.21 \pm 0.13 \pm 0.30$ \\
$0.8-1.0$ & $4.51 \pm 0.22 \pm 0.48$ & $2.051 \pm 0.011 \pm 0.044$ & $2.35 \pm 0.12 \pm 0.25$ \\
$1.0-1.4$ & $2.07 \pm 0.13 \pm 0.33$ & $1.031 \pm 0.006 \pm 0.032$ & $2.18 \pm 0.13 \pm 0.42$ \\
$1.4-2.0$ & $0.91 \pm 0.06 \pm 0.15$ & $0.3876 \pm 0.0030 \pm 0.0095$ & $2.62 \pm 0.17 \pm 0.46$ \\
$2.0-3.0$ & $0.16 \pm 0.03 \pm 0.10$ & $0.1074 \pm 0.0012 \pm 0.0037$ & $1.71 \pm 0.28 \pm 0.82$ \\
$3.0-4.0$ & $0.0167 \pm 0.0084 \pm 0.019$ & $0.01837 \pm 0.00047 \pm 0.00098$ & $1.11 \pm 0.56 \pm 0.81$ \\
\hline
\end{tabular}
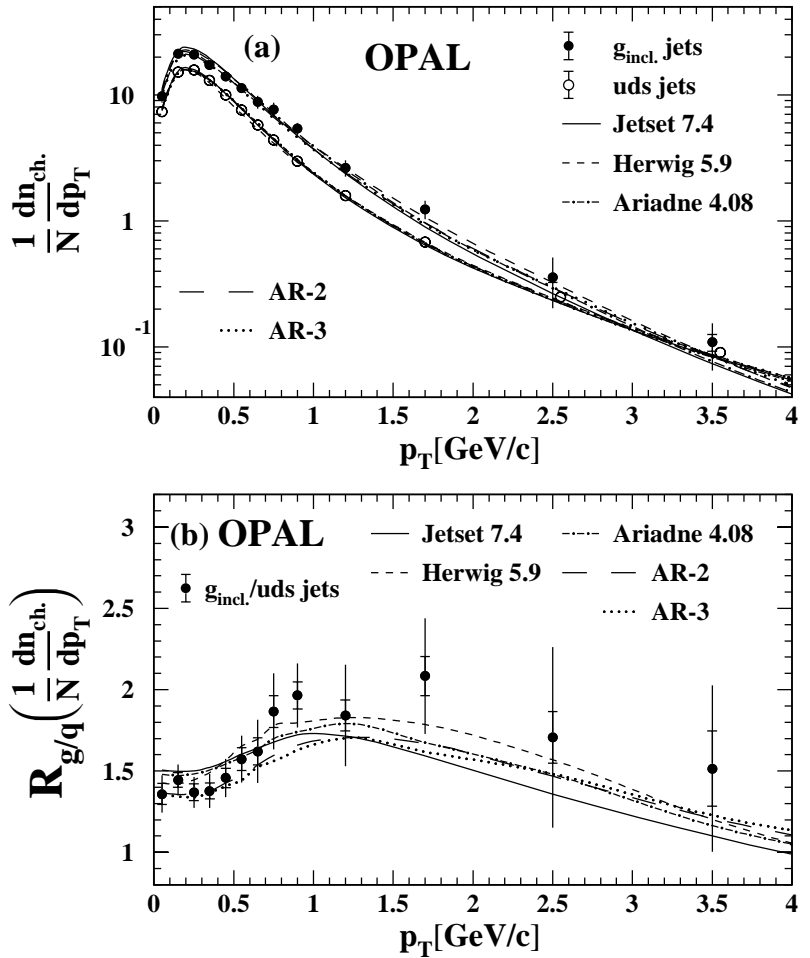

Fig. 8. a Corrected distributions of charged particle transverse momentum with respect to the jet axis, $p_{\mathrm{T}}$, for $40.1 \mathrm{GeV} \mathrm{g}_{\text {incl. }}$. gluon jets and $45.6 \mathrm{GeV}$ uds quark jets. $\mathbf{b}$ The ratio of the gluon to quark jet $p_{\mathrm{T}}$ distributions for $40.1 \mathrm{GeV}$ jets. The total uncertainties are shown by vertical lines. The experimental statistical uncertainties are indicated by small horizontal bars. (The uncertainties are too small to be seen for the uds jets.) The predictions of various parton shower Monte Carlo event generators are also shown. These data are tabulated in Table 4
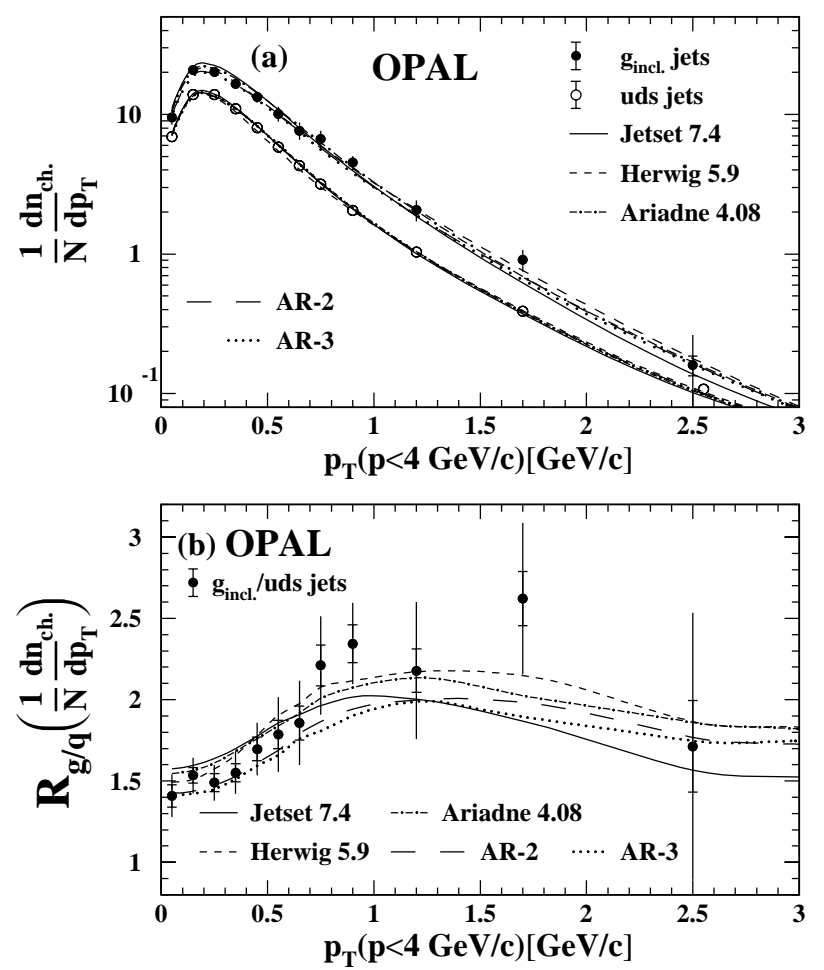

Fig. 9. a Corrected distributions of charged particle transverse momentum with respect to the jet axis for particles with momentum $p<4 \mathrm{GeV} / c, p_{\mathrm{T}}(p<4 \mathrm{GeV} / c)$, for $40.1 \mathrm{GeV} g_{\text {incl. }}$ gluon jets and $45.6 \mathrm{GeV}$ uds quark jets. $\mathbf{b}$ The ratio of the gluon to quark jet $p_{\mathrm{T}}(p<4 \mathrm{GeV} / c)$ distributions for $40.1 \mathrm{GeV}$ jets. The total uncertainties are shown by vertical lines. The experimental statistical uncertainties are indicated by small horizontal bars. (The uncertainties are too small to be seen for the uds jets.) The predictions of various parton shower Monte Carlo event generators are also shown. These data are tabulated in Table 5 

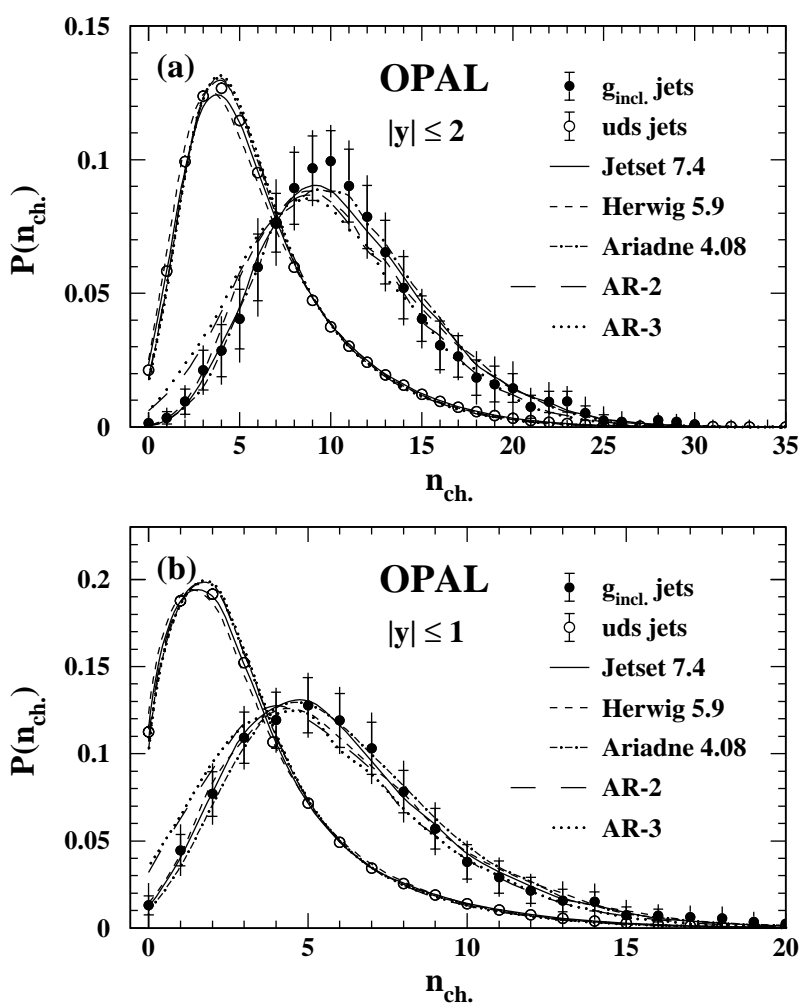

Fig. 10. Corrected distributions of charged particle multiplicity in the rapidity intervals $\mathbf{a}|y| \leq 2$ and $\mathbf{b}|y| \leq 1$ for $40.1 \mathrm{GeV}$ $\mathrm{g}_{\text {incl. }}$ gluon jets and $45.6 \mathrm{GeV}$ uds quark jets. The total uncertainties are shown by vertical lines. The experimental statistical uncertainties are indicated by small horizontal bars. (The statistical uncertainties are too small to be seen for the uds jets.) The data are correlated between bins. The predictions of various parton shower Monte Carlo event generators are also shown. These data are tabulated in Tables 6 and 7

totic QCD result. Specifically, we determine the Herwig prediction for $r_{\mathrm{ch} .} .(|y| \leq 1)$ and the corresponding result $r(|y| \leq 1)$ at the parton level. To determine these ratios, we use event hemispheres in Herwig gg and uds events. At the hadron level, Herwig predicts $r_{\text {ch. }}(|y| \leq 1)$ to be 1.92 for $E_{\text {jet }}=40.1 \mathrm{GeV}$ and 2.18 for $E_{\text {jet }}=5 \mathrm{TeV}$ (the statistical uncertainties of the Monte Carlo results are negligible). At the parton level, the corresponding results for $r(|y| \leq 1)$ are 2.06 and 2.25. The hadron level result for $40.1 \mathrm{GeV}$ is in good agreement with our measurement (relation (3)). The parton level result for $5 \mathrm{TeV}$ jets yields precisely the QCD asymptotic value of 2.25 demonstrating that our experimental variable $r_{\text {ch. }}(|y| \leq 1)$ does indeed correspond to $r$ as it is defined analytically. Thus, our data are consistent with the QCD prediction. The hadronization corrections, given by the ratios of the parton to the hadron level Monte Carlo results, are 1.07 for $E_{\text {jet }}=40.1 \mathrm{GeV}$ and 1.03 for $E_{\text {jet }}=5 \mathrm{TeV}$. The corrections for finite energy, given by the ratios of the Herwig results at $5 \mathrm{TeV}$ to those at $40.1 \mathrm{GeV}$, are 1.14 at the hadron level and 1.09 at the parton level. We conclude that the difference between our measurement $\left(r_{\mathrm{ch} .}(|y| \leq 1) \approx 1.92\right)$ and the QCD pre-

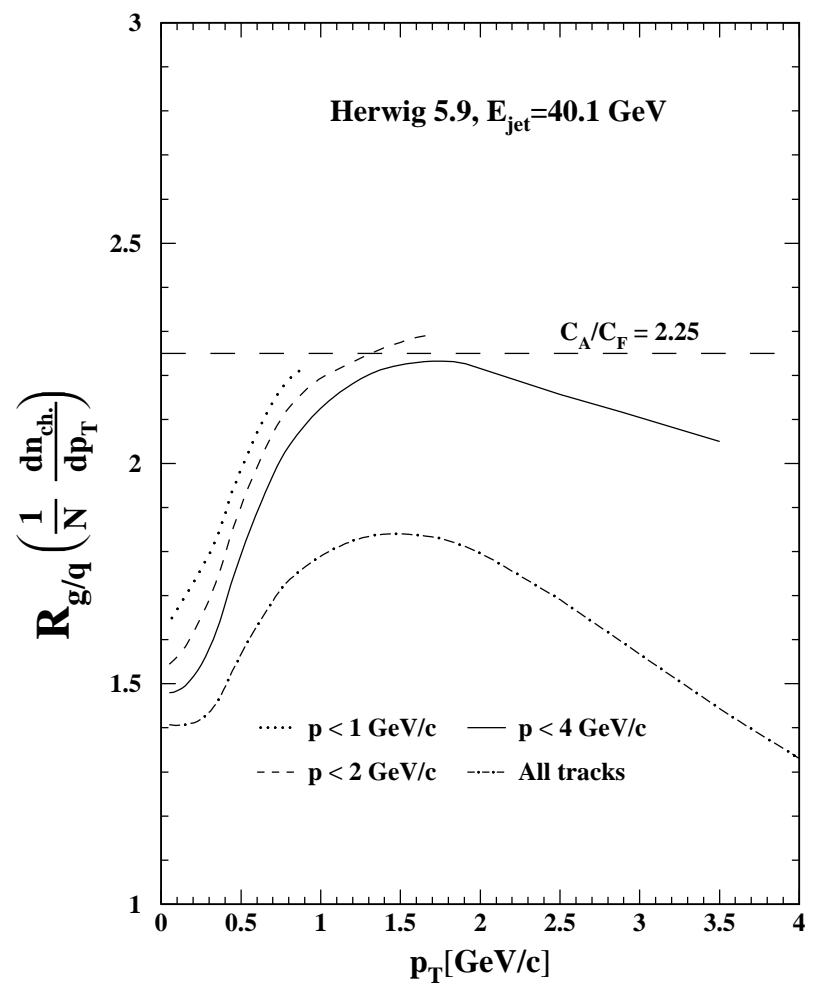

Fig. 11. The prediction of the Herwig Monte Carlo event generator for the ratio of the transverse momentum distributions between $40.1 \mathrm{GeV}$ gluon and $40.1 \mathrm{GeV}$ uds quark jets, for all charged particles and for charged particles with momentum $p$ below 4,2 and $1 \mathrm{GeV} / c$

diction $(r=2.25)$ can mostly be explained by the effects of finite energy.

\subsection{Mean multiplicity ratio of soft particles at large $p_{\mathrm{T}}$}

It was recently noted [36] that the multiplicity ratio of gluon to quark jets should exhibit a value near the full asymptotic prediction of $\mathrm{C}_{\mathrm{A}} / \mathrm{C}_{\mathrm{F}}=2.25$, even at the finite energies of LEP, if soft particles with large transverse momenta to the jet axes are considered: soft gluons at large angles to the jet axes in gg or q⿳亠口冋 events are emitted coherently, with a coupling strength proportional to the effective color charge of the parton initiating the jet, given by $\mathrm{C}_{\mathrm{A}}$ for gluon jets and $\mathrm{C}_{\mathrm{F}}$ for quark jets. We therefore examined the predictions of the Herwig Monte Carlo for the ratio of the $p_{\mathrm{T}}$ distributions of gluon to quark jets for soft charged hadrons defined by $p<4.0 \mathrm{GeV} / c$. We used hemispheres in Herwig gg and uds events to define the jets, with $E_{\text {jet }}=40.1 \mathrm{GeV}$ to correspond to our data. We chose Herwig for this study for the reasons outlined in Sect. 9.1. The results are shown by the solid curve in Fig. 11. For values of $p_{\mathrm{T}}$ below about $0.2 \mathrm{GeV} / c$, the ratio of the gluon to quark jet multiplicity is predicted to have a value near 1.5 . This ratio increases to approximately 2.25 for $p_{\mathrm{T}} \approx 1 \mathrm{GeV} / c$ and remains near this value for larger $p_{\mathrm{T}}$. Analogous results are obtained for soft hadrons defined by $p<2.0 \mathrm{GeV} / c$ and $p<1.0 \mathrm{GeV} / c$ (dashed and 

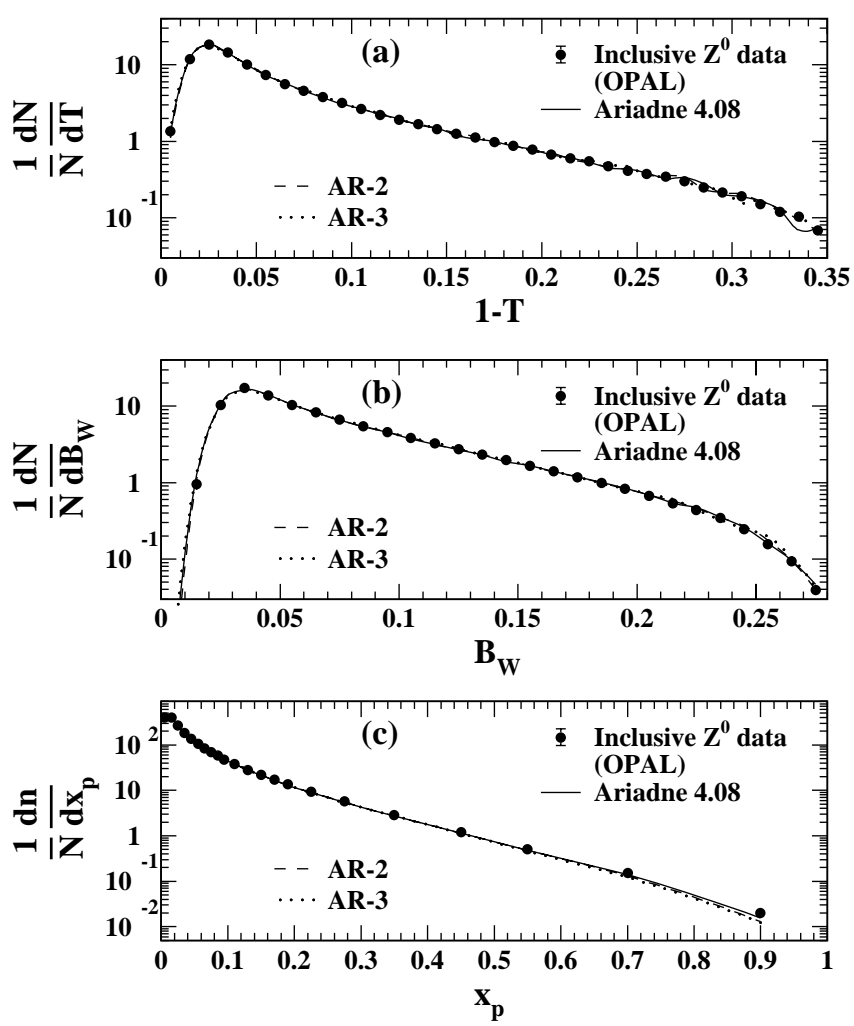

Fig. 12a-c. Comparison of the predictions of the standard version of Ariadne and of the two versions of Ariadne with color reconnection to inclusive $\mathrm{Z}^{0}$ event data: a 1-T [41], with $\mathrm{T}$ the thrust, $\mathbf{b}$ jet broadening variable $\mathrm{B}_{\mathrm{W}}$ [41], and $\mathbf{c}$ scaled particle momentum $x_{p}=2 p / E_{\text {c.m. }}$. [32]. The total uncertainties, with statistical and systematic terms added in quadrature, are too small to be visible

dotted curves), i.e. the curves reach values near 2.25 for $p_{\mathrm{T}}$ values above about $1.0 \mathrm{GeV} / c$ irrespective of the precise definition of soft particles. If all charged particles are selected, and not just soft ones, the predicted multiplicity ratio reaches a maximum of only about 1.85 , however (dash-dotted curve). The results of Fig. 11 suggest that the multiplicity ratio of soft hadrons at large $p_{\mathrm{T}}$ effectively yields a measurement of $\mathrm{C}_{\mathrm{A}} / \mathrm{C}_{\mathrm{F}}$ at LEP [36]. It is for this reason that we include the $p_{\mathrm{T}}$ spectrum of charged particles with $p<4.0 \mathrm{GeV} / c$ in our study (see Sect. 5). The experimental data for this distribution, $p_{\mathrm{T}}(p<4 \mathrm{GeV} / c)$, were previously presented in Fig. 9 and Table 5 .

As a measure of the soft particle multiplicity at large $p_{\mathrm{T}}$, we integrate the $p_{\mathrm{T}}(p<4 \mathrm{GeV} / c)$ distribution between $p_{\mathrm{T}}$ values of 0.8 and $3.0 \mathrm{GeV} / c$ : this range is chosen on the basis of Fig. 11, as the region where the ratio of the gluon to quark jet multiplicity is predicted to approximately equal $\mathrm{C}_{\mathrm{A}} / \mathrm{C}_{\mathrm{F}}$. We choose the upper limit of integration to be $p_{\mathrm{T}}=3.0 \mathrm{GeV} / c$ to avoid the region near the kinematic boundary at $p_{\mathrm{T}}=4 \mathrm{GeV} / c$ (in practice this makes little difference because of the small statistical weight of particles with $p_{\mathrm{T}}>3 \mathrm{GeV} / c$ ). Our measurement of this quantity, $\left(r_{\mathrm{ch}}\right)_{0.8<p_{\mathrm{T}}<3 \mathrm{GeV} / c}^{p<4 \mathrm{GeV} / c}$, is:

$\left(r_{\text {ch. }}\right)_{0.8<p_{\mathrm{T}}<3 \mathrm{GeV} / c}^{p<4 \mathrm{GeV} / c}=2.29 \pm 0.09$ (stat.) \pm 0.15 (syst.) (4)
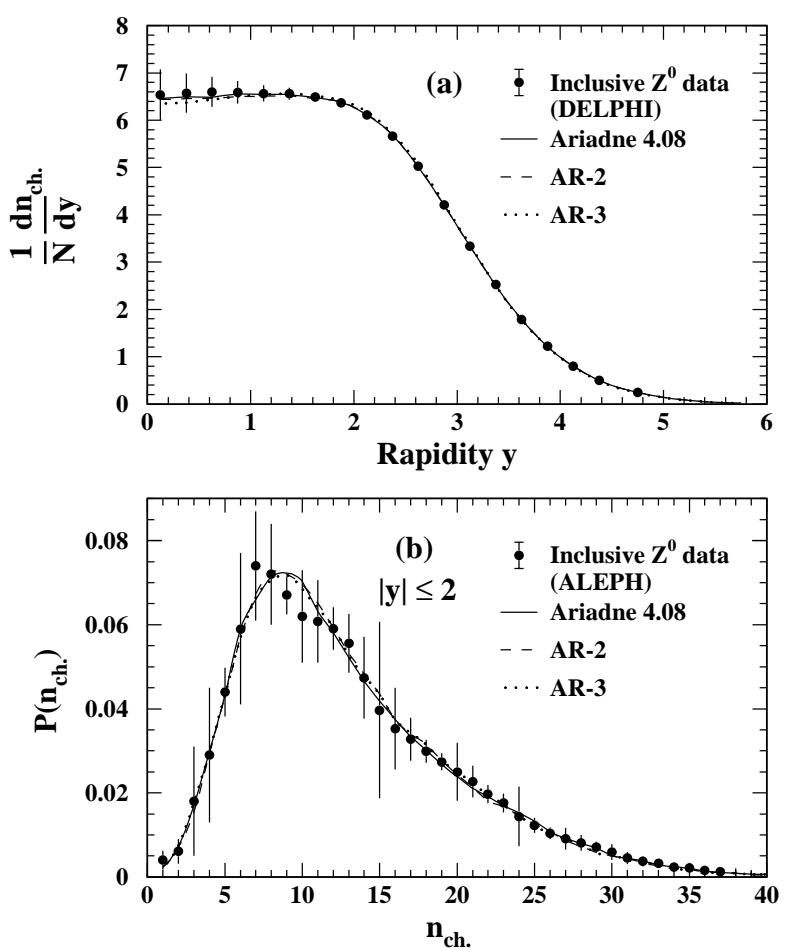

Fig. 13a,b. Comparison of the predictions of the standard version of Ariadne and of the two versions of Ariadne with color reconnection to inclusive $Z^{0}$ event data: a rapidity $y$ with respect to the sphericity axis [42], and $\mathbf{b}$ charged particle multiplicity in the interval $|y| \leq 2$ [43]. The total uncertainties are shown by the vertical lines, with statistical and systematic terms added in quadrature

This result is summarized in Table 8. The corresponding results from Herwig at the hadron and parton levels are 2.16 and 2.09 with negligible statistical uncertainties. The hadron level result agrees with our measurement (4) to within the experimental uncertainties. For $5 \mathrm{TeV}$ jets, Herwig predicts 2.23 and 2.25 at the hadron and parton levels: the latter result equals the QCD asymptotic prediction for $r$, demonstrating the correspondence between the variable (4) and $r$ as it is defined analytically, similar to the variable $r_{\text {ch. }}(|y| \leq 1)$ considered in Sect. 9.1. The ratio (4) has smaller predicted corrections than the variable considered in Sect. 9.1: the hadronization correction for $\left(r_{\text {ch. }}\right)_{0.8<p_{\mathrm{T}}<3 \mathrm{GeV} / c}^{p<4 \mathrm{GeV} / c}$ at $40.1 \mathrm{GeV}$ is 0.97 (compared to 1.07 for $r_{\text {ch. }}(|y| \leq 1)$ ), while the correction for finite energy between $40.1 \mathrm{GeV}$ and $5 \mathrm{TeV}$ hadron jets is 1.03 (compared to 1.14). Thus the ratio of soft hadron multiplicities between gluon and quark jets at large $p_{\mathrm{T}}$ does indeed yield a value consistent with $\mathrm{C}_{\mathrm{A}} / \mathrm{C}_{\mathrm{F}}$ to within the uncertainties, even at the finite energies of LEP, as predicted in [36].

For completeness, we also report the results we obtain for the gluon to quark jet multiplicity ratio using different choices for the intervals of $p$ and $p_{\mathrm{T}}$. For $p<2 \mathrm{GeV} / c$ (rather than $p<4 \mathrm{GeV} / c)$ we obtain $\left(r_{\text {ch. }}\right)_{p_{\mathrm{T}}>0.8 \mathrm{GeV} / c}^{p<2 \mathrm{GeV} / c}=$ $2.32 \pm 0.12$ (stat.) \pm 0.14 (syst.). For $0.8<p_{\mathrm{T}}<2.0 \mathrm{GeV} / c$ (rather than $\left.0.8<p_{\mathrm{T}}<3.0 \mathrm{GeV} / c\right)$ we obtain $\left(r_{\text {ch. }}\right)_{0.8<p_{\mathrm{T}}<2 \mathrm{GeV} / c}^{p<4 \mathrm{GeV} / c}=2.33 \pm 0.08$ (stat.) \pm 0.16 (syst.). 

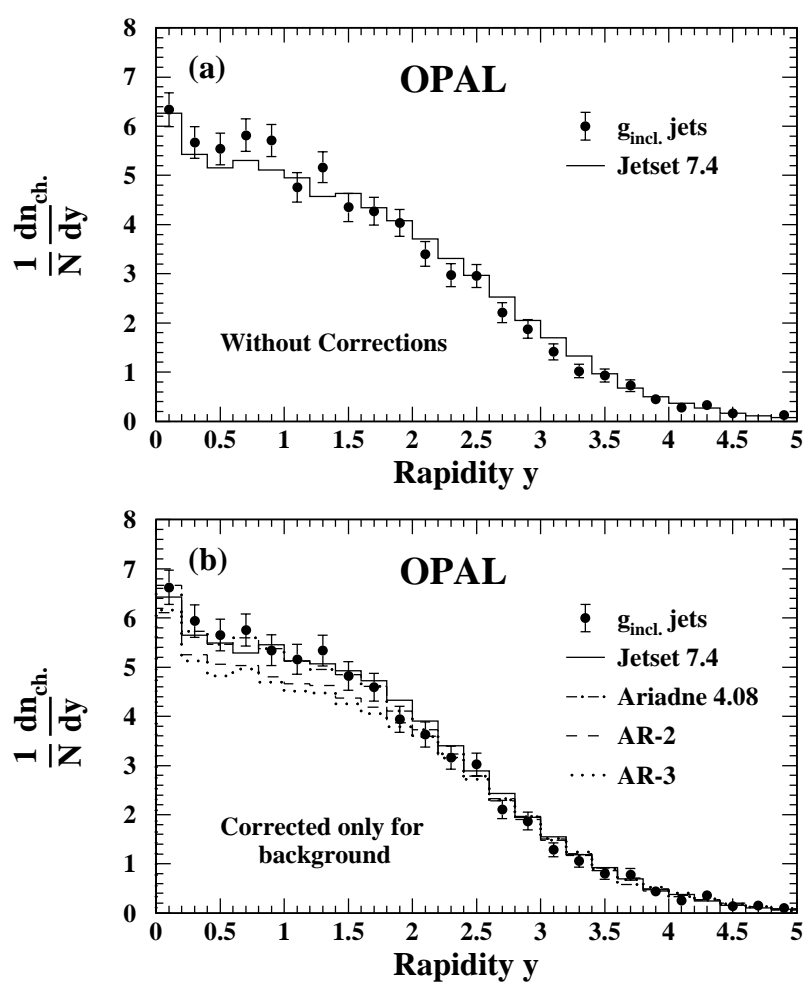

Fig. 14a,b. The distribution of charged particle rapidity for $40.1 \mathrm{GeV}$ g incl. gluon jets: a uncorrected distribution, i.e. at the level which includes background, detector acceptance and resolution, secondary interactions, initial-state radiation, and the experimental track and cluster selection criteria, and $\mathbf{b}$ distribution corrected for background only. The uncertainties are statistical

These results are very similar to the result (4) reported above.

\subsection{Fragmentation function}

Another striking feature of our results is the much softer fragmentation function of gluon jets compared to quark jets (Fig. 6). That gluon jets have a softer fragmentation function than quark jets has already been well established. These earlier studies either employed a jet finder to define the gluon jets $[19,22,37]$ or extracted the gluon jet fragmentation function using measurements of the longitudinal and transverse fragmentation functions in $\mathrm{e}^{+} \mathrm{e}^{-}$ annihilations $[26,38]$. Unlike the earlier studies based on jet finders, we employ theoretically well defined jets. Unlike the earlier studies utilizing the longitudinal and transverse fragmentation functions, the energy scale of our jets is well defined. Therefore our results have more theoretical meaning than these previous ones.

\section{Monte Carlo predictions}

Figs. 5-10 include the hadron level predictions of Jetset, Herwig and Ariadne. The Monte Carlo results for $\mathrm{g}_{\text {incl. }}$
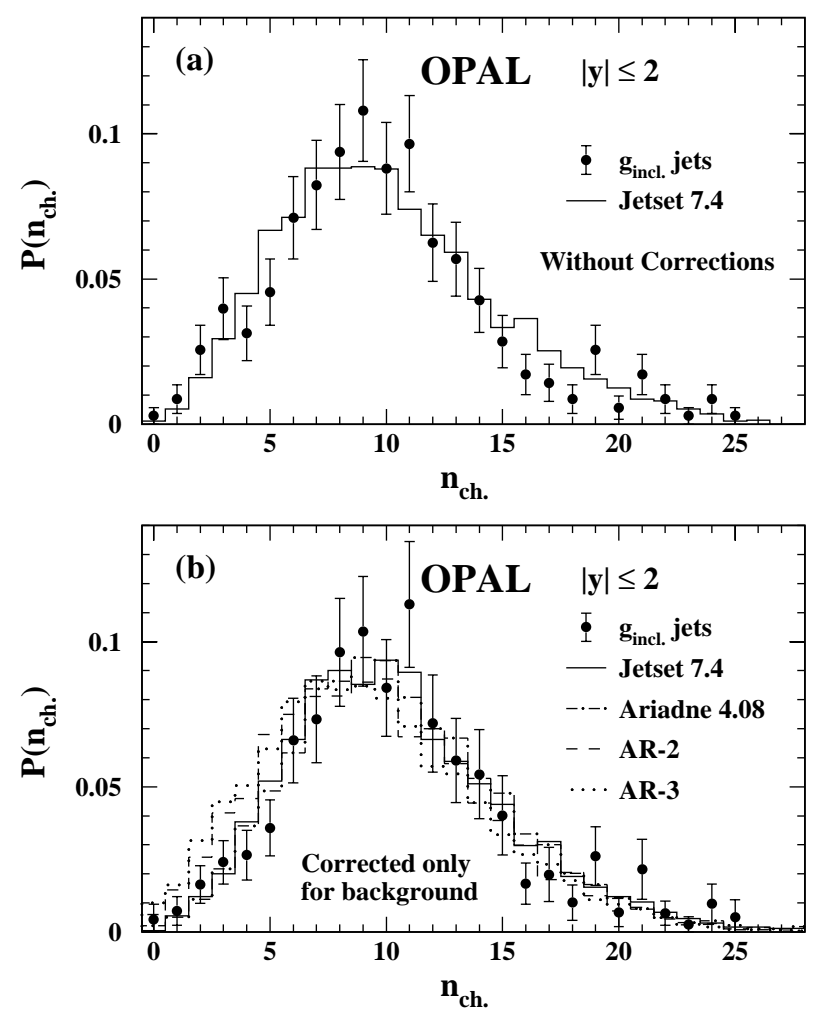

Fig. 15a,b. The distribution of charged particle multiplicity in the rapidity interval $|y| \leq 2$ for $40.1 \mathrm{GeV} \mathrm{g}_{\text {incl. gluon }}$ jets: a uncorrected distribution, i.e. at the level which includes background, detector acceptance and resolution, secondary interactions, initial-state radiation, and the experimental track and cluster selection criteria, and $\mathbf{b}$ distribution corrected for background only. The uncertainties are statistical

jets are obtained using parton level information in the manner described in Sect. 3. The results for Ariadne are shown both with and without the effects of color reconnection. For the standard version of Ariadne, i.e. the version without reconnection, we use the parameter values given in [28] with the following modification: the value of the " $a$ " parameter ${ }^{10}$ controlling the hardness of the fragmentation function is increased from 0.40 to 0.52 to obtain a better description of $\left\langle n_{\text {ch. }}\right\rangle$ in inclusive multihadronic $\mathrm{Z}^{0}$ events. These parameters provide a substantial improvement in the description of our uds jet data compared to the default parameters. We examine two versions of the Ariadne model with reconnection, referred to here as AR-2 and AR-3 to conform to our previous usage [39]. In the AR-2 model $^{11}$, gluons are not subject to reconnection (see Sect. 11) unless their energies are below a cutoff. ${ }^{12}$ For our study, this cutoff is set to $2 \mathrm{GeV}[10]$. We generate events using the parameters in [28] except for the $a$ parameter which is adjusted to 0.65 to obtain an accurate description of $\left\langle n_{\text {ch. }}\right\rangle$ in inclusive $\mathrm{Z}^{0}$ events. In the AR-3 model ${ }^{13}$,

${ }_{10}$ Given by the Jetset Monte Carlo parameter PARJ(41)

11 Enabled by setting the parameter $\operatorname{MSTA}(35)=2$

12 Given by the parameter PARA(28)

13 Enabled by setting the parameter $\operatorname{MSTA}(35)=3$ 
Table 6. Charged particle multiplicity distributions, expressed in per cent (\%), of $40.1 \mathrm{GeV} \mathrm{g}_{\text {incl. }}$ gluon jets and $45.6 \mathrm{GeV}$ uds quark jets, in the rapidity interval $|y| \leq 2$. The mean values $\left\langle n_{\text {ch. }}(|y| \leq 2)\right\rangle$ are also given. The first uncertainty is statistical and the second is systematic. The data are correlated between bins. These data are displayed in Fig. 10a

\begin{tabular}{|c|c|c|}
\hline$n_{\text {ch. }}(|y| \leq 2)$ & $\mathrm{g}_{\text {incl. }}$ gluon jet & uds quark jet \\
\hline 0 & $0.15 \pm 0.01 \pm 0.26$ & $2.13 \pm 0.04 \pm 0.13$ \\
\hline 1 & $0.34 \pm 0.23 \pm 0.31$ & $5.83 \pm 0.07 \pm 0.17$ \\
\hline 2 & $0.94 \pm 0.47 \pm 0.40$ & $9.93 \pm 0.08 \pm 0.22$ \\
\hline 3 & $2.13 \pm 0.74 \pm 0.56$ & $12.37 \pm 0.10 \pm 0.21$ \\
\hline 4 & $2.86 \pm 0.97 \pm 0.83$ & $12.67 \pm 0.10 \pm 0.21$ \\
\hline 5 & $4.0 \pm 1.1 \pm 1.2$ & $11.47 \pm 0.09 \pm 0.19$ \\
\hline 6 & $6.0 \pm 1.2 \pm 1.4$ & $9.52 \pm 0.10 \pm 0.18$ \\
\hline 7 & $7.6 \pm 1.1 \pm 1.2$ & $7.63 \pm 0.07 \pm 0.18$ \\
\hline 8 & $8.9 \pm 1.3 \pm 0.83$ & $5.98 \pm 0.08 \pm 0.17$ \\
\hline 9 & $9.7 \pm 1.2 \pm 0.8$ & $4.74 \pm 0.07 \pm 0.16$ \\
\hline 10 & $9.9 \pm 1.1 \pm 0.8$ & $3.75 \pm 0.05 \pm 0.13$ \\
\hline 11 & $9.0 \pm 1.4 \pm 1.0$ & $3.02 \pm 0.06 \pm 0.10$ \\
\hline 12 & $7.9 \pm 1.2 \pm 1.0$ & $2.418 \pm 0.042 \pm 0.072$ \\
\hline 13 & $6.5 \pm 1.2 \pm 0.9$ & $1.937 \pm 0.045 \pm 0.052$ \\
\hline 14 & $5.2 \pm 1.2 \pm 0.8$ & $1.561 \pm 0.034 \pm 0.043$ \\
\hline 15 & $4.04 \pm 0.85 \pm 0.72$ & $1.218 \pm 0.036 \pm 0.038$ \\
\hline 16 & $3.05 \pm 0.90 \pm 0.55$ & $0.957 \pm 0.029 \pm 0.038$ \\
\hline 17 & $2.64 \pm 0.77 \pm 0.44$ & $0.734 \pm 0.027 \pm 0.036$ \\
\hline 18 & $1.84 \pm 0.66 \pm 0.74$ & $0.564 \pm 0.022 \pm 0.033$ \\
\hline 19 & $1.60 \pm 0.68 \pm 0.78$ & $0.423 \pm 0.019 \pm 0.027$ \\
\hline 20 & $1.45 \pm 0.53 \pm 0.86$ & $0.322 \pm 0.017 \pm 0.020$ \\
\hline 21 & $0.75 \pm 0.43 \pm 0.55$ & $0.241 \pm 0.014 \pm 0.014$ \\
\hline 22 & $0.93 \pm 0.40 \pm 0.62$ & $0.181 \pm 0.011 \pm 0.011$ \\
\hline 23 & $0.95 \pm 0.39 \pm 0.42$ & $0.132 \pm 0.010 \pm 0.010$ \\
\hline 24 & $0.52 \pm 0.27 \pm 0.56$ & $0.0894 \pm 0.0090 \pm 0.0073$ \\
\hline 25 & $0.24 \pm 0.21 \pm 0.48$ & $0.0640 \pm 0.0081 \pm 0.0053$ \\
\hline 26 & $0.19 \pm 0.15 \pm 0.38$ & $0.0440 \pm 0.0059 \pm 0.0047$ \\
\hline 27 & - & $0.0267 \pm 0.0047 \pm 0.0052$ \\
\hline 28 & $0.25 \pm 0.09 \pm 0.27$ & $0.0207 \pm 0.0039 \pm 0.0056$ \\
\hline 29 & $0.19 \pm 0.06 \pm 0.30$ & $0.0144 \pm 0.0027 \pm 0.0040$ \\
\hline 30 & $0.09 \pm 0.05 \pm 0.13$ & $0.0084 \pm 0.0023 \pm 0.0025$ \\
\hline 31 & - & $0.0036 \pm 0.0020 \pm 0.0024$ \\
\hline 32 & - & $0.0031 \pm 0.0014 \pm 0.0016$ \\
\hline 33 & - & $0.0007 \pm 0.0011 \pm 0.0015$ \\
\hline 34 & - & - \\
\hline 35 & - & $0.00079 \pm 0.00056 \pm 0.00065$ \\
\hline 36 & - & $0.00031 \pm 0.00034 \pm 0.00045$ \\
\hline$\left\langle n_{\text {ch. }}(|y| \leq 2)\right\rangle$ & $10.83 \pm 0.20 \pm 0.41$ & $6.085 \pm 0.013 \pm 0.071$ \\
\hline
\end{tabular}

gluons of all energies are subject to reconnection. For this model we use the parameters in [28] except with the $a$ parameter set to 0.58 to describe $\left\langle n_{\text {ch. }}\right\rangle$ in inclusive $\mathrm{Z}^{0}$ events. The results for the mean charged particle multiplicity in inclusive hadronic $\mathrm{Z}^{0}$ events are 20.9, 20.9 and 21.0 for our tuned versions of AR-2, AR-3 and the standard version of Ariadne, respectively, in agreement with the measured value of $21.0 \pm 0.2$ [26]-[28].

The three versions of Ariadne yield very similar descriptions of standard measures of event properties in in-
Table 7. Charged particle multiplicity distributions, expressed in per cent (\%), of $40.1 \mathrm{GeV} \mathrm{g}_{\text {incl. }}$ gluon jets and $45.6 \mathrm{GeV}$ uds quark jets, in the rapidity interval $|y| \leq 1$. The mean values $\left\langle n_{\text {ch. }}(|y| \leq 1)\right\rangle$ are also given. The first uncertainty is statistical and the second is systematic. The data are correlated between bins. These data are displayed in Fig. 10b

\begin{tabular}{|c|c|c|}
\hline$n_{\text {ch. }}(|y| \leq 1)$ & $\mathrm{g}_{\text {incl. }}$ gluon jet & uds quark jet \\
\hline 0 & $1.3 \pm 0.5 \pm 1.1$ & $11.25 \pm 0.09 \pm 0.41$ \\
\hline 1 & $4.5 \pm 0.9 \pm 1.2$ & $18.77 \pm 0.11 \pm 0.33$ \\
\hline 2 & $7.7 \pm 1.3 \pm 1.2$ & $19.16 \pm 0.12 \pm 0.32$ \\
\hline 3 & $10.9 \pm 1.5 \pm 1.1$ & $15.23 \pm 0.10 \pm 0.26$ \\
\hline 4 & $11.9 \pm 1.6 \pm 0.9$ & $10.66 \pm 0.09 \pm 0.26$ \\
\hline 5 & $12.8 \pm 1.6 \pm 1.0$ & $7.16 \pm 0.07 \pm 0.22$ \\
\hline 6 & $11.9 \pm 1.6 \pm 1.1$ & $4.92 \pm 0.06 \pm 0.20$ \\
\hline 7 & $10.3 \pm 1.5 \pm 1.4$ & $3.44 \pm 0.06 \pm 0.16$ \\
\hline 8 & $7.8 \pm 1.2 \pm 1.3$ & $2.56 \pm 0.04 \pm 0.12$ \\
\hline 9 & $5.7 \pm 1.2 \pm 1.0$ & $1.897 \pm 0.042 \pm 0.079$ \\
\hline 10 & $3.79 \pm 0.98 \pm 0.71$ & $1.395 \pm 0.040 \pm 0.064$ \\
\hline 11 & $2.92 \pm 0.92 \pm 0.64$ & $1.032 \pm 0.032 \pm 0.052$ \\
\hline 12 & $2.16 \pm 0.74 \pm 0.60$ & $0.750 \pm 0.024 \pm 0.039$ \\
\hline 13 & $1.57 \pm 0.65 \pm 0.91$ & $0.546 \pm 0.023 \pm 0.025$ \\
\hline 14 & $1.52 \pm 0.54 \pm 0.89$ & $0.395 \pm 0.021 \pm 0.014$ \\
\hline 15 & $0.72 \pm 0.48 \pm 0.77$ & $0.273 \pm 0.017 \pm 0.010$ \\
\hline 16 & $0.71 \pm 0.37 \pm 0.53$ & $0.194 \pm 0.013 \pm 0.008$ \\
\hline 17 & $0.64 \pm 0.25 \pm 0.59$ & $0.128 \pm 0.011 \pm 0.006$ \\
\hline 18 & $0.56 \pm 0.21 \pm 0.64$ & $0.0847 \pm 0.0087 \pm 0.0049$ \\
\hline 19 & $0.36 \pm 0.21 \pm 0.54$ & $0.0555 \pm 0.0072 \pm 0.0041$ \\
\hline 20 & $0.25 \pm 0.12 \pm 0.28$ & $0.0376 \pm 0.0047 \pm 0.0036$ \\
\hline 21 & - & $0.0204 \pm 0.0045 \pm 0.0038$ \\
\hline 22 & - & $0.0136 \pm 0.0036 \pm 0.0033$ \\
\hline 23 & - & $0.0101 \pm 0.0026 \pm 0.0041$ \\
\hline 24 & - & $0.0058 \pm 0.0019 \pm 0.0041$ \\
\hline 25 & - & $0.0036 \pm 0.0015 \pm 0.0041$ \\
\hline 26 & - & $0.0022 \pm 0.0011 \pm 0.0023$ \\
\hline$\left\langle n_{\text {ch. }}(|y| \leq 1)\right\rangle$ & $6.14 \pm 0.15 \pm 0.36$ & $3.333 \pm 0.010 \pm 0.046$ \\
\hline
\end{tabular}

clusive $\mathrm{Z}^{0}$ multihadronic events, such as thrust, sphericity, aplanarity (see e.g. [40] for a definition of these variables) and the quantities defined in Sect. 5. Their overall descriptions of inclusive $\mathrm{Z}^{0}$ data are good. To illustrate these points, we calculated the $\chi^{2}$ values between the predictions of the models and the measured distributions of thrust $\mathrm{T}$ [41], jet broadening variable $\mathrm{B}_{\mathrm{W}}$ [41], scaled particle momentum $x_{p}=2 p / E_{\text {c.m. }}$. [32], rapidity with respect to the sphericity axis [42], and charged particle multiplicity in the rapidity interval $|y| \leq 2$ [43], for inclusive hadronic $\mathrm{Z}^{0}$ events. (Note that there are correlations between these variables and between different bins of some of the distributions.) These last three variables are chosen because of their similarity to distributions studied in this paper (Sect. 5). The total $\chi^{2}$ values for 152 bins of data are 293 for AR-2, 241 for AR-3, and 290 for the standard version of Ariadne. For purposes of comparison, the corre- 
Table 8. The ratios $r_{\mathrm{ch}}$. of the mean charged particle multiplicity between $40.1 \mathrm{GeV} \mathrm{g}_{\text {incl. gluon }}$ jets and $40.1 \mathrm{GeV}$ uds quark jets for full phase space, for restricted rapidity intervals $|y| \leq 2$ and $|y| \leq 1$, and for soft particles at large transverse momentum $p_{\mathrm{T}}$ with respect to the jet axis, defined by $p<4 \mathrm{GeV} / c$ and $0.8<p_{\mathrm{T}}<3 \mathrm{GeV} / c$, with $p$ the particle momentum. The results are given for the data and for QCD Monte Carlo programs. The corresponding results at the parton level are given in parentheses for the standard QCD models and for a special version of Jetset with $\mathrm{C}_{\mathrm{A}}=\mathrm{C}_{\mathrm{F}}=4 / 3$. For the data, the first uncertainty is statistical and the second is systematic

\begin{tabular}{ccccc}
\hline & $r_{\text {ch. }}$ & $r_{\text {ch. }}(|y| \leq 2)$ & $r_{\text {ch. }}(|y| \leq 1)$ & $\left(r_{\text {ch. }}\right)_{0.8<p_{\mathrm{T}}<3 \mathrm{GeV} / c}^{p<4 \mathrm{GeV} / c}$ \\
\hline OPAL data & $\begin{array}{c}1.514 \pm 0.019 \\
\pm 0.034\end{array}$ & $\begin{array}{c}1.852 \pm 0.034 \\
\pm 0.077\end{array}$ & $\begin{array}{c}1.919 \pm 0.047 \\
\pm 0.095\end{array}$ & $\begin{array}{c}2.29 \pm 0.09 \\
\pm 0.15\end{array}$ \\
\hline Standard QCD models: & & & \\
Herwig & $1.54(1.56)$ & $1.85(1.97)$ & $1.92(2.06)$ & $2.16(2.09)$ \\
Jetset & $1.54(1.35)$ & $1.84(1.61)$ & $1.88(1.62)$ & $1.93(1.50)$ \\
Ariadne & $1.55(1.46)$ & $1.89(1.77)$ & $1.97(1.81)$ & $2.07(1.81)$ \\
\hline Jetset $\mathrm{C}_{\mathrm{A}}=\mathrm{C}_{\mathrm{F}}=4 / 3$ & $1.38(1.03)$ & $1.54(1.06)$ & $1.51(1.04)$ & $1.31(1.00)$ \\
\hline Reconnected QCD models: & & & \\
AR-2 & 1.43 & 1.72 & 1.78 & 1.95 \\
AR-3 & 1.42 & 1.69 & 1.75 & 1.92 \\
\hline
\end{tabular}

sponding result from Jetset is 322 . These $\chi^{2}$ values are determined using both statistical and systematic uncertainties, with no account for correlations. They are intended to be used only as a relative measure of the description of inclusive $\mathrm{e}^{+} \mathrm{e}^{-}$data by the different Monte Carlo models. A good description does not imply that $\chi^{2}$ should approximately equal the number of data bins. In Figs. 12 and 13, the predictions of the three versions of Ariadne are shown in comparison to the inclusive $\mathrm{Z}^{0}$ event measurements. The predictions of the three variants of Ariadne are seen to be virtually indistinguishable from each other and in good agreement with the data.

From Figs. 5-10, it is seen that the Monte Carlo simulations provide a good description of the gluon jet properties, with the exception of the AR-2 and AR-3 color reconnection models whose predictions for $\mathrm{g}_{\text {incl. }}$ jets are discussed in the next section. All the models provide a reasonable description of the uds jet measurements. The predictions of the AR-2 and AR-3 models for the uds jet properties are essentially identical to those of the standard version of Ariadne.

The Monte Carlo predictions for the multiplicity ratios $r_{\text {ch. }}, r_{\text {ch. }}(|y| \leq 2), r_{\text {ch. }}(|y| \leq 1)$ and $\left(r_{\text {ch. }}\right)_{0.8<p_{\mathrm{T}}<3 \mathrm{GeV} / c}^{p<4 \mathrm{GeV} / c}$ are given in Table 8 . In addition to the results for charged hadrons, the results are given at the parton level (in parentheses) for Herwig, Jetset and the standard version of Ariadne. The hadron level predictions are in general agreement with the data, with the exception of the Jetset prediction for $\left(r_{\text {ch. }}\right)_{0.8<p_{\mathrm{T}}<3 \mathrm{GeV} / c}^{p<4 \mathrm{GeV} / c}$ which is about 2 standard deviations of the total experimental uncertainty below the measurement (this is possibly related to the failure of Jetset to yield the QCD asymptotic result $\mathrm{r}=\mathrm{C}_{\mathrm{A}} / \mathrm{C}_{\mathrm{F}}$ at large jet energies [5]). Also included in Table 8 are the predictions of a special version of Jetset in which the color factor $\mathrm{C}_{\mathrm{A}}$ governing gluon jet evolution has been set equal to the factor $\mathrm{C}_{\mathrm{F}}=4 / 3$ governing quark jet evolution. The parton level results with $\mathrm{C}_{\mathrm{A}}=\mathrm{C}_{\mathrm{F}}=4 / 3$ essentially equal unity, i.e. the ratio values vary from 1.00 to 1.06 , in contrast to the parton level predictions of the standard version of Jetset which range from 1.35 to 1.62, emphasizing the sensitivity of the multiplicity ratios to the value of $\mathrm{C}_{\mathrm{A}} / \mathrm{C}_{\mathrm{F}}$. At the hadron level, the multiplicity ratios obtained using this special version of Jetset vary between 1.31 and 1.54: the results at the hadron level are not expected to equal unity, even with $\mathrm{C}_{\mathrm{A}}=\mathrm{C}_{\mathrm{F}}$, because the Jetset hadronization model treats quarks and gluons differently. ${ }^{14}$

\section{Test of a model for color reconnection}

Most implementations of QCD, including those in the standard versions of Jetset, Herwig and Ariadne, are based on the so-called large $\mathrm{N}_{\mathrm{c}}$ approximation, with $\mathrm{N}_{\mathrm{c}}$ the number of colors. In this approximation, the manner in which partons are connected to form an overall color singlet is uniquely specified. For example, in $\mathrm{Z}^{0} \rightarrow \mathrm{q} \overline{\mathrm{q} g g}$ events, in which two gluons gg are radiated from a quark $q$ and antiquark $\overline{\mathrm{q}}$ produced from a $\mathrm{Z}^{0}$ decay, the quark is colorconnected to one of the gluons (e.g. connected by a color flux tube, which is modelled as a cluster chain or string in the Monte Carlo programs), this first gluon is colorconnected to the second gluon, and the second gluon is color-connected to the antiquark $\overline{\mathrm{q}}$. Thus, the entire event consists of a single color singlet system. This color singlet hadronizes, with hadrons appearing preferentially in the regions spanned by the color flux.

In the large $\mathrm{N}_{\mathrm{c}}$ approximation, interference terms of relative order $1 / \mathrm{N}_{\mathrm{c}}^{2}$ are ignored. If these interference terms are included to obtain predictions valid beyond the large

\footnotetext{
14 Quarks are attached to a single string segment in the Lund model of hadronization [44] implemented in Jetset, whereas gluons are attached to two string segments
} 
Table 9. Probability, measured in per cent, for a $\mathrm{g}_{\text {incl. jet }}$ to have four or fewer charged particles with rapidity $|y| \leq 2$. The results are given for the data and for QCD Monte Carlo programs. For the data, the first uncertainty is statistical and the second is systematic

\begin{tabular}{cc}
\hline & $\begin{array}{c}\text { Probability for } n_{\text {ch. }}(|y| \leq 2) \leq 4 \\
\text { in } g_{\text {incl. }} \text { jets }\end{array}$ \\
\hline OPAL data & $6.42 \pm 1.30 \pm 1.65 \%$ \\
\hline Standard QCD models: & $6.0 \%$ \\
Jetset & $7.1 \%$ \\
Herwig & $5.4 \%$ \\
Ariadne & \\
\hline Reconnected QCD models: & $11.2 \%$ \\
AR-2 & $12.2 \%$ \\
AR-3
\end{tabular}

$\mathrm{N}_{\mathrm{c}}$ approximation, the manner in which partons are connected to each other is no longer specified uniquely. For example, in $\mathrm{Z}^{0} \rightarrow \mathrm{q} \overline{\mathrm{q}} \mathrm{gg}$ events, the possibility that the $\mathrm{q}$ and $\bar{q}$ form a color singlet by themselves, with the two gluons gg forming a separate color singlet, occurs with probability $^{15} 1 /\left(\mathrm{N}_{\mathrm{c}}^{2}-1\right)$ relative to the "normal" situation described in the previous paragraph. The possibility of defining the color singlets in this latter manner is an example of what is called color reconnection. Color reconnection can affect events at both the perturbative and non-perturbative levels: its effects at the perturbative level are expected to be small, however, in comparison to its effects at the non-perturbative level [45]. To assess the effects of color reconnection at the non-perturbative level, several models for reconnection have been implemented in non-standard versions of QCD Monte Carlo event generators. With the exception of the VNI model [46] discussed in [39], none of these models has been subjected to a stringent test. Color reconnection has been a topic of recent interest due to the possibility that reconnected diagrams could measurably affect the reconstructed $\mathrm{W}$ boson mass in $\mathrm{e}^{+} \mathrm{e}^{-} \rightarrow$ $\mathrm{W}^{+} \mathrm{W}^{-} \rightarrow \mathrm{q}_{1} \overline{\mathrm{q}}_{2} \mathrm{q}_{3} \overline{\mathrm{q}}_{4}$ events recorded at LEP-2 [8].

In general, color reconnection can have a significant influence on the energy and angular distributions of hadrons in an event since the color flux spans different regions of phase space compared to normal color connection. Since the standard Monte Carlo programs provide a good description of the general properties of inclusive $\mathrm{Z}^{0}$ hadronic events, it can be inferred that the overall effect of reconnection is small. It is nonetheless possible that the effects of reconnection are sizable in special classes of events such as the $\mathrm{e}^{+} \mathrm{e}^{-} \rightarrow \mathrm{q} \overline{\mathrm{q}} \mathrm{g}_{\text {incl. }}$ events studied here. Indeed, it has been suggested $[8,9]$ that this class of events - with a pure system of gluons recoiling against a quark-antiquark system in the opposite hemisphere - can provide a sensitive test for the presence of reconnection phenomena. In the following, we use our data to test the Ariadne color re-

\footnotetext{
15 In addition, dynamical effects can lead to a further sup-
} pression of these "reconnected" terms connection models AR-2 and AR-3 presented in Sect. 10. We choose these models for our study because they provide good descriptions of inclusive $\mathrm{Z}^{0}$ data, as discussed in Sect. 10, and thus represent realistic models of nature (unlike the VNI model of reconnection which does not describe the basic properties of $\mathrm{W}^{+} \mathrm{W}^{-}$events [39]).

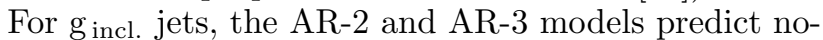
ticeably fewer particles at small rapidities and energies, and noticeably more particles at large rapidities and energies, than are observed in either the data or standard QCD programs (see Figs. 5 and 6). Furthermore, these two models predict a downwards shift of about one unit in the $g_{\text {incl. }}$ charged particle multiplicity distributions compared to the data or the standard QCD programs (see Fig. 10). Thus, our $g_{\text {incl. data are indeed sensitive to color }}$ reconnection effects. To test the sensitivity of the models' predictions to their parameters, we varied the values of the main parameters ${ }^{16}$ within the uncertainties given in [28]: the predictions of the models remained virtually unchanged. We note that the predictions of the AR-2 and AR-3 models are in much more serious disagreement with our data, compared to the disagreement seen in Figs. 5, 6 and 10, if the default Ariadne parameter set is used rather than the parameter sets described in Sect. 10.

As a quantitative measure of the difference between our data and the predictions of Ariadne with reconnection, we performed two related tests. For the first test, we compared the values of $r_{\mathrm{ch}}$. The results are summarized in the bottom portion of Table 8: $r_{\mathrm{ch}}$. in full phase space is predicted to be 1.43 and 1.42 by the AR-2 and AR-3 models, which are 2.2 and 2.4 standard deviations of the total experimental uncertainty below the measured value of $1.514 \pm 0.039$ (stat.+syst.). For the second test, we compared the probability, measured in per cent, for a $\mathrm{g}_{\text {incl. }}$ jet to have four or fewer charged particles with $|y| \leq 2$ (a comparison of this nature is suggested in [9]). To determine these probabilities, we integrated the $\mathrm{g}_{\text {incl. distributions }}$ in Fig. 10a from $n_{\text {ch. }}=0$ to $n_{\text {ch. }}=4$. The upper limit of $n_{\mathrm{ch}}=4$ is chosen because it yields the maximum deviation of the predictions of AR-2 and AR-3 with respect to the standard version of Ariadne, using the statistical uncertainties of the data, compared to other choices. The results are given in Table 9. The AR-2 and AR-3 models predict $11.2 \%$ and $12.2 \%$ for these probabilities, in disagreement with the measured value of $6.4 \pm 2.1$ (stat. + syst.) $\%$ by 2.3 and 2.8 standard deviations, respectively. In contrast, the standard QCD programs reproduce the experimental result well (Table 9).

The results of the previous paragraph are based on fully corrected data, emphasizing the absolute measurement of gluon jet multiplicity. By examining the gluon jet properties at the level which includes detector acceptance and resolution and the experimental selection criteria, it is possible to emphasize the relative difference between data and model since factors like the experimental track and cluster definitions are common to both. Such a comparison is presented in Figs. 14 and 15 for rapidity

\footnotetext{
${ }_{16}$ Specifically, PARA(1), PARA(3), PARJ(21) and $\operatorname{PARJ}(42)$
} 
and charged particle multiplicity with $|y| \leq 2$. In Figs. 14a and $15 \mathrm{a}$, the predictions of Jetset including detector simulation and the same analysis procedures as are applied to the data are shown in comparison to the experimental measurements without corrections. Jetset is seen to reproduce the data well, without significant systematic deviations. In Figs. 14b and 15b, the predictions of Jetset and the three versions of Ariadne are shown after including detector simulation and the experimental selection criteria of Sects. 2 and 3, except that the $q$ and $\bar{q}$ for the $g_{\text {incl. jet }}$ selection are identified using parton level information as described in Sect. 3 rather than using displaced secondary vertices: we do not employ this latter method to obtain the model predictions for Figs. $14 \mathrm{~b}$ and $15 \mathrm{~b}$ due to a lack of sufficient Ariadne Monte Carlo event statistics which include simulation of the detector. The data in Figs. 14b and $15 \mathrm{~b}$ have been corrected for the $18 \%$ background to

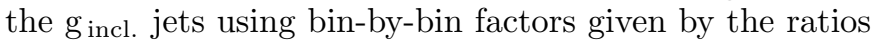
of the Jetset predictions in Figs. $14 \mathrm{~b}$ and $15 \mathrm{~b}$ to those in Figs. 14a and 15a, respectively. Thus the data and model results in Figs. 14b and 15b correspond to pure gluon jets which have not been corrected for detector acceptance and resolution. By comparing the relative differences between Jetset and the data in Fig. 14a and b, and similarly in Fig. 15a and b, it is seen that no significant bias is introduced in the gluon jet measurements by applying the corrections for background.

The discrepancies of AR-2 and AR-3 with the data, noted above in connection with the fully corrected results (cf. Figs. 5 and 10), are clearly visible in Figs. 14b and 15b: these two models predict significantly fewer particles at small rapidities $(|y| \leq 2)$ than are observed experimentally. In contrast, Jetset and the version of Ariadne without reconnection are seen to describe the data well. The $\chi^{2}$ values between the data and models are 26, 17, 45 and 63 for Jetset, Ariadne, AR-2 and AR-3, for the 25 bins of data shown in Fig. 14b. The corresponding results for the 10 bins of data with $|y| \leq 2$ are $6,3,29$ and 43. Integrating the distributions of Fig. $15 \mathrm{~b}$ between $n_{\mathrm{ch} .}=0$ and $n_{\text {ch. }}=5$ (i.e. similar to the test presented in Table 9 for the fully corrected data), we obtain $12.9 \pm 0.6$ (stat.) $\%$ for Jetset, $12.5 \pm 0.6$ (stat.) $\%$ for Ariadne, $20.1 \pm 0.6$ (stat.) $\%$ for AR-2 and $21.6 \pm 0.6$ (stat.) $\%$ for AR-3, compared to the measured value of $11.4 \pm 1.8$ (stat.)\%: this represents a discrepancy between data and model of 4.7 standard deviations for AR-2 and of 5.4 standard deviations for AR-3. The value $n_{\mathrm{ch}}=5$ is chosen as the upper limit of integration for this last result because it yields the maximum deviation of the predictions of AR-2 and AR-3 with respect to the standard version of Ariadne, at the level including detector simulation, compared to other choices.

On the basis of the results presented above, we conclude that the AR-2 and AR-3 color reconnection models implemented in Ariadne are disfavored. This result may be of some benefit in the assessment of systematic uncertainties for the $\mathrm{W}$ boson mass measurement at LEP-2.

\section{Summary and conclusions}

In this paper, we have presented experimental measurements of the properties of gluon and light flavored (uds) quark jets. The jets are defined by inclusive sums over the particles in $g_{\text {incl. }}$ and $u d s$ event hemispheres, with the $\mathrm{g}_{\text {incl. }}$ gluon jet opposite to a hemisphere containing two identified quark jets in $\mathrm{e}^{+} \mathrm{e}^{-}$annihilations (the quark jets

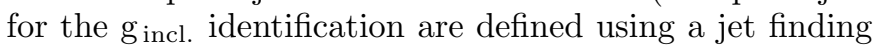
algorithm). These inclusive definitions are in close correspondence to the definition of jets used for QCD calculations, based on the production of virtual gluon and quark jet pairs, gg and $q \bar{q}$, from a color singlet point source. We present the distributions of rapidity, scaled energy, the logarithm of the momentum, transverse momentum with respect to the jet axis, and multiplicity in restricted intervals of rapidity, for charged particles in the gluon and quark jets. Our results for gluon jets are almost entirely independent of the choice of a jet finding algorithm, a unique feature of our analysis compared to other studies of high energy $\left(E_{\text {jet }}>5 \mathrm{GeV}\right)$ gluon jets. The energy of the jets in our study is about $40 \mathrm{GeV}$.

We determine the ratio, $r_{\mathrm{ch}}$, of the mean gluon to quark jet charged particle multiplicity for particles with rapidities $|y| \leq 1$ to be $r_{\text {ch. }}(|y| \leq 1)=1.919 \pm 0.047$ (stat.) \pm 0.095 (syst.). The corresponding ratio for soft particles at large transverse momentum, defined by $p<4 \mathrm{GeV} / c$ and $0.8<p_{\mathrm{T}}<3.0 \mathrm{GeV} / c$, is found to be $\left(r_{\mathrm{ch}}\right)_{0.8<p_{\mathrm{T}}<3 \mathrm{GeV} / c}^{p<4 \mathrm{GeV} / c}$ $=2.29 \pm 0.09$ (stat.) \pm 0.15 (syst.). Our measurement of this last quantity is motivated by the prediction that the multiplicity difference between gluon and quark jets for soft particles emitted at large angles to the jet axes approximately equals the ratio of $\mathrm{QCD}$ color factors, $\mathrm{C}_{\mathrm{A}} / \mathrm{C}_{\mathrm{F}}=$ 2.25 , even at the finite energies of LEP [36]. Using the Herwig Monte Carlo, we verify that our results are consistent with the QCD prediction that the mean multiplicities of soft particles in gluon and quark jets differ by a factor of $r=\mathrm{C}_{\mathrm{A}} / \mathrm{C}_{\mathrm{F}}=2.25$ [34], once the effects of hadronization and finite energy have been considered. Because our experimental definition of jets corresponds to the theoretical one, our results are the most direct test of this QCD prediction to date.

Further, we use our data to perform the most stringent test to date of the model of color reconnection [10] implemented in the Ariadne Monte Carlo. We find that this model does not describe our gluon jet measurements accurately. This result may be of some utility in assessing the systematic uncertainty associated with color reconnection in the determination of the $\mathrm{W}$ boson mass from $\mathrm{e}^{+} \mathrm{e}^{-} \rightarrow \mathrm{W}^{+} \mathrm{W}^{-} \rightarrow \mathrm{q}_{1} \overline{\mathrm{q}}_{2} \mathrm{q}_{3} \overline{\mathrm{q}}_{4}$ events recorded at LEP-2.

Acknowledgements. We thank Stan Brodsky, Valery Khoze and Wolfgang Ochs for valuable discussions, and Torbjörn Sjöstrand for help in implementing the version of Jetset with $\mathrm{C}_{\mathrm{A}}=\mathrm{C}_{\mathrm{F}}$ mentioned in Sect. 10. We particularly wish to thank the SL Division for the efficient operation of the LEP accelerator and for their continuing close cooperation with our experimental group. We thank our colleagues from CEA, DAPNIA/SPP, CE-Saclay for their efforts over the years on the time-of-flight 
and trigger systems which we continue to use. In addition to the support staff at our own institutions we are pleased to acknowledge the Department of Energy, USA, National Science Foundation, USA, Particle Physics and Astronomy Research Council, UK, Natural Sciences and Engineering Research Council, Canada, Israel Science Foundation, administered by the Israel Academy of Science and Humanities, Minerva Gesellschaft, Benoziyo Center for High Energy Physics, Japanese Ministry of Education, Science and Culture (the Monbusho) and a grant under the Monbusho International Science Research Program, Japanese Society for the Promotion of Science (JSPS), German Israeli Bi-national Science Foundation (GIF), Bundesministerium für Bildung, Wissenschaft, Forschung und Technologie, Germany, National Research Council of Canada, Research Corporation, USA, Hungarian Foundation for Scientific Research, OTKA T-016660, T023793 and OTKA F-023259.

\section{References}

1. TASSO Collaboration, R. Brandelik et al., Phys. Lett. B86 (1979) 243; PLUTO Collaboration, C. Berger et al., Phys. Lett. B86 (1979) 418; MARK J Collaboration, D.P. Barber et al., Phys. Rev. Lett. 43 (1979) 830; JADE Collaboration, W. Bartel et al., Phys. Lett. B91 (1980) 142.

2. CLEO Collaboration, M.S. Alam et al., Phys. Rev. D46 (1992) 4822; CLEO Collaboration, M.S. Alam et al., Phys. Rev. D56 (1997) 17.

3. J.W. Gary, Phys. Rev. D49 (1994) 4503.

4. Yu.L. Dokshitzer, V.A. Khoze and S.I. Troyan, Sov. J. Nucl. Phys. 47 (1988) 881.

5. OPAL Collaboration, G. Alexander et al., Phys. Lett. B388 (1996) 659.

6. OPAL Collaboration, K. Ackerstaff et al., Eur. Phys. J. C1 (1998) 479.

7. G. Gustafson, U. Pettersson and P.M. Zerwas, Phys. Lett. B209 (1988) 90.

8. Z. Kunszt, W.J. Stirling et al., Physics at LEP-2, CERN 96-01, Vol. 1, p.141, eds. G. Altarelli, T. Sjöstrand and F. Zwirner, Geneva 1996.

9. C. Friberg, G. Gustafson and J. Häkkinen, Nucl. Phys. B490 (1997) 289.

10. L. Lönnblad, Z. Phys. C70 (1996) 107.

11. L. Lönnblad, Comp. Phys. Comm. 71 (1992) 15.

12. OPAL Collaboration, K. Ahmet et al., Nucl. Instr. and Meth. A305 (1991) 275.

13. P.P. Allport et al., Nucl. Instr. and Meth. A346 (1994) 476.

14. OPAL Collaboration, G. Alexander et al., Z. Phys. C52 (1991) 175.

15. S. Brandt et al., Phys. Lett. 12 (1964) 57;

E. Fahri, Phys. Rev. Lett. 39 (1977) 1587

16. S. Catani et al., Phys. Lett. B269 (1991) 432.

17. See, for example, The LEP Collaborations, ALEPH, DELPHI, L3 and OPAL, the LEP Electroweak Working Group, and the SLD Heavy Flavor Group, CERNPPE/97-154.

18. DELPHI Collaboration, P. Abreu et al., Phys. Lett. B405 (1997) 202;

ALEPH Collaboration, R. Barate et al., Phys. Lett. B434 (1998) 437.
19. OPAL Collaboration, R. Akers et al., Z. Phys. C68 (1995) 179.

20. T. Sjöstrand, Comp. Phys. Comm. 82 (1994) 74.

21. J. Allison et al., Nucl. Instr. and Meth. A317 (1992) 47.

22. OPAL Collaboration, P.D. Acton et al., Z. Phys. C58 (1993) 387.

23. OPAL Collaboration, G. Alexander et al., Z. Phys. C69 (1996) 543.

24. J.D. Bjorken and S.J. Brodsky, Phys. Rev. D1 (1970) 1416 ;

SLAC-LBL Collaboration, G. Hanson et al., Phys. Rev. Lett. 35 (1975) 1609.

25. G. Marchesini, B.R. Webber et al., Comp. Phys. Comm. 67 (1992) 465.

26. OPAL Collaboration, R. Akers et al., Z. Phys. C68 (1995) 203.

27. DELPHI Collaboration, P. Abreu et al., Z. Phys. C73 (1996) 11.

28. ALEPH Collaboration, R. Barate et al., Phys. Rep. 294 (1998) 1.

29. JADE Collaboration, W. Bartel et al., Z. Phys. C33 (1986) 23.

30. OPAL Collaboration, R. Akers et al., Z. Phys. C63 (1994) 197.

31. OPAL Collaboration, P.D. Acton et al., Z. Phys. C53 (1992) 539.

32. OPAL Collaboration, K. Ackerstaff et al., CERN-EP/98089.

33. S. Lupia and W. Ochs, Phys. Lett. B418 (1998) 214; P. Eden and G. Gustafson, JHEP 09(1998)015.

34. S.J. Brodsky and J. Gunion, Phys. Rev. Lett. 37 (1976) 402;

K. Konishi, A. Ukawa and G. Veneziano, Phys. Lett. B78 (1978) 243.

35. S.J. Brodsky, private communication.

36. V.A. Khoze, S. Lupia and W. Ochs, Eur. Phys. J. C5 (1998) 77.

37. OPAL Collaboration, G. Alexander et al., Phys. Lett. B265 (1991) 462;

DELPHI Collaboration, P. Abreu et al., Z. Phys. C70 (1996) 179 ;

ALEPH Collaboration, D. Buskulic et al., Phys. Lett. B384 (1996) 353.

38. ALEPH Collaboration, D. Buskulic et al., Phys. Lett. B357 (1995) 487;

DELPHI Collaboration, P. Abreu et al., Eur. Phys. J. C6 (1999) 19.

39. OPAL Collaboration, G. Abbiendi et al., CERN-EP/98196.

40. OPAL Collaboration, M.Z. Akrawy et al., Z. Phys. C47 (1990) 505.

41. OPAL Collaboration, P.D. Acton et al., Z. Phys. C59 (1993) 1

42. DELPHI Collaboration, P. Abreu et al., Z. Phys. C73 (1996) 11.

43. ALEPH Collaboration, D. Buskulic et al., Z. Phys. C69 (1995) 15.

44. B. Andersson et al., Phys. Rep. 97 (1983) 31.

45. V.A. Khoze and T. Sjöstrand, Phys. Rev. Lett. 72 (1994) 28; Z. Phys. C62 (1994) 281.

46. K. Geiger, Comp. Phys. Comm. 104 (1997) 70. 